\title{
1 Stratification of the Gut Microbiota Composition Landscape Across the 2 Alzheimer's Disease Continuum in a Turkish Cohort
}

Süleyman Yıldırım*1,2, Özkan Ufuk Nalbantoğlu*3,4, Abdulahad Bayraktar5, Fatma Betül Ercan ${ }^{6}$, Aycan Gündoğdu ${ }^{4,7}$, Halil Aziz Velioğlu²,6, Mehmet Fatih Göl ${ }^{7}$, Ayten Ekinci Soylu , Fatma Koç ${ }^{1 \&}$, Ezgi

Aslan Gürpınar ${ }^{7}$, Kübra Sogukkanlı Kadak ${ }^{6}$, Muzaffer Arıkan², Adil Mardinoğlu5,8, Mehmet Koçak ${ }^{9}$, Emel

Köseoğlu\#,10, Lütfü Hanoğlu $u^{\#, 11}$

8

1. Department of Medical Microbiology, Istanbul Medipol University International School of Medicine, Istanbul, Turkey

2. Regenerative and Restorative Medicine Research Center (REMER); Research Institute for Health Sciences and Technologies (SABITA), Istanbul Medipol University, Istanbul, Turkey.

3. Department of Computer Engineering, Erciyes University, Kayseri, Turkey

4. Genome and Stem Cell Center (GenKok), Erciyes University, Kayseri, Turkey

5. Centre for Host-Microbiome Interactions, Faculty of Dentistry, Oral \& Craniofacial Sciences, King's College London, London, SE1 9RT, UK

6. Graduate Program in Neuroscience, Istanbul Medipol University International School of Medicine, Istanbul, Turkey

7. Department of Microbiology and Clinical Microbiology, Erciyes University School of Medicine, Kayseri, Turkey

8. Science for Life Laboratory, KTH - Royal Institute of Technology, Stockholm, SE-17121, Sweden

9. Department of Preventive Medicine, University of Tennessee Health Science Center, Memphis, TN, United States of America

10. Department of Neurology, School of Medicine, Erciyes University, Kayseri, Turkey

11. Department of Neurology, School of Medicine, Istanbul Medipol University, Istanbul, Turkey.

Running title: Gut Microbiota Stratification in Alzheimer's Disease

Key Words: Gut microbiome, Alzheimer's Diseases, 16S rRNA, Stratification

${ }^{*}$ Equal contribution.

\#Corresponding authors:

Email addresses:

Dr. Süleyman Yildirim

suleymanyildirim@medipol.edu.tr

Dr. Emel Koseoglu

emelk@erciyes.edu.tr

Dr. Lutfu Hanoglu

$\underline{\text { Ihanoglu@medipol.edu.tr }}$

${ }^{\&}$ Current address: APC Microbiome Ireland, University College Cork, Cork T12 YT20, Ireland 


\section{ABSTRACT}

53 Alzheimer's disease $(A D)$ is a heterogeneous neurodegenerative disorder that spans over a continuum with multiple phases including preclinical, mild cognitive impairment, and dementia. Unlike most other chronic diseases there are limited number of human studies reporting on AD gut microbiota in the literature. These published studies suggest that the gut microbiota of $A D$ continuum patients varies considerably throughout the disease stages, raising expectations for existence of multiple microbiota community types. However, the community types of AD gut microbiota were not systematically investigated before, leaving important research gap for dietbased intervention studies and recently initiated precision nutrition approaches aiming at

61 stratifying patients into distinct dietary subgroups. Here, we comprehensively assessed the

62 community types of gut microbiota across the AD continuum. We analyze $16 \mathrm{~S}$ rRNA amplicon

63 sequencing of stool samples from 27 mild cognitive patients, $47 \mathrm{AD}$, and 51 non-demented control

64 subjects using tools compatible with compositional nature of microbiota. To characterize gut 65 microbiota community types, we applied multiple machine learning techniques including 66 partitioning around the medoid clustering, fitting probabilistic Dirichlet mixture model, Latent

67 Dirichlet Allocation model, and performed topological data analysis for population scale 68 microbiome stratification based on Mapper algorithm. These four distinct techniques all converge 69 on Prevotella and Bacteroides partitioning of the gut microbiota across AD continuum while some 70 methods provided fine scale resolution in partitioning the community landscape. The Signature 71 taxa and neuropsychometric parameters together robustly classify the heterogenous groups 72 within the cohort. Our results provide a framework for precision nutrition approaches and dietbased intervention studies targeting AD cohorts. 
IMPORTANCE

78 The prevalence of $A D$ worldwide is estimated to reach 131 million by 2050 . Most disease

79 modifying treatments and drug trials have failed due partly to the heterogeneous and complex

80 nature of the disease. Unlike other neurodegenerative diseases gut microbiota of AD patients is

81 poorly studied. Recently initiated ambitious precision nutrition initiative or other diet-based

82 interventions can potentially be more effective if the heterogeneous disease such as $A D$ is

83 deconstructed into multiple strata allowing for better identification of biomarkers across narrower

84 patient population for improved results. Because gut microbiota is inherently integral part of the

85 nutritional interventions there is unmet need for microbiota-informed stratification of AD clinical

86 cohorts in nutritional studies. Our study fills in this gap and draws attention to the need for

87 microbiota stratification as one of the essential steps for precision nutrition interventions. We

demonstrate that while Prevotella and Bacteroides clusters are the consensus partitions the newly developed probabilistic methods can provide fine scale resolution in partitioning the AD gut microbiome landscape.

92 Key words: Alzheimer's Disease, Gut microbiota, Machine learning, Stratification, Dirichlet,

93 Topological data analysis

\section{INTRODUCTION}

97 Alzheimer's Disease $(A D)$ is the most common form of dementia worldwide and its prevalence is estimated to reach 131 million by 2050 [1]. AD spans over a continuum starting with the non-

99 symptomatic pre-clinical stage and advancing through the spectrum of clinical stages. These

100 stages are dashed with distinct pathophysiological states [2], namely the amyloid-tau101 neuroinflammation axis. The clinical continuum entails mild memory loss and/or cognitive 
102 impairments (mild cognitive impairment, $\mathrm{MCl}$ due to $\mathrm{AD}$ ) and trajectories for function leading to

103 memory problems besides cognitive impairment (dementia phase); and finally complete loss of

104 independent functioning towards the end stage [3]. Moreover, The Alzheimer's dementia phase

105 is further broken down into the stages of mild, moderate and severe, thereby making AD a

106 complex and highly heterogenous disease.

108 Traditionally, pathogenesis of AD is attributed to extracellular aggregation of amyloid- $\beta$-peptides

$109(\mathrm{~A} \beta)$ in senile plaques and intracellular depositions of hyperphosphorylated tau that forms

110 neurofibrillary tangles [4]. Although numerous clinical trials based on the amyloid postulates have

111 been attempted virtually all of them have failed [5]. The unsettlingly consistent failure of clinical

112 trials targeting single target amyloid pathways prompted researchers to refine the amyloid

113 hypothesis [6] and even extend it to periphery [7]. Recently, a group of AD researchers asserted

114 that infectious agents reach and remain dormant in the central nervous system (CNS) and

115 undergo reactivation during aging, sparking cascades of inflammation, induce $A \beta$, and ultimately

116 neuronal degeneration [8]. Chronic inflammation in CNS mediated by microglial toxicity as well

117 as systemic inflammation in the periphery is widely recognized in $A D$ and linked to amyloid

118 cascade hypothesis in animal experiments $[9,10]$. None of the drugs available today for

119 Alzheimer's dementia slow or stop the damage and destruction of neurons [11]. Intervention at

120 different points along the Alzheimer's continuum should therefore be multimodal and involve

121 targeting neuropathology in brain, systemic inflammation in the body, and metabolic processes in

122 the periphery that escalate the disease in brain [12]. Non-pharmacologic, targeted, personalized,

123 and multimodal disease modifying interventions in $A D$, including diet and lifestyle changes to

124 optimize metabolic parameters has recently been under investigation [13-16].

126 A growing body of evidence suggest that human gut microbiota is strongly associated with human

127 metabolic processes in all organs including brain [17] and implicated in neuroinflammation via 
128 brain-gut axis [18]. Gut microbes across animal models influence CNS by modulation of

129 neuroimmune function, sensory neuronal signaling, and metabolic activity [19]. Several studies

130 using transgenic animal model of $A D$ reported gut microbiota alterations (see [19]) but these

131 animal models poorly mirror human AD. Unexpectedly, only a few human clinical studies on AD

132 were reported in the literature [20-28]. Of these studies, gut microbiota associated metabolites

133 such elevated Trimethylamine N-oxide (TMAO) in CSF [26] and altered bile acids profile [28] were

134 directly implicated in AD dementia. Importantly, dietary pattern of AD patients is at the center of

135 the precision medicine approaches [29]. Also, diet is one of the most important factors modulating

136 gut microbiota-based active metabolites. Disease modifying approaches involving diet should

137 therefore consider microbiota in AD. Indeed, a recent study [23] tested the impact of a modified

138 Mediterranean ketogenic diet on gut microbiome composition and demonstrated that the diet can

139 modulate the gut microbiome and metabolites in association with improved AD biomarkers in

140 CSF. These published studies, however, did not comprehensively investigate AD microbiota

141 subclusters across the disease continuum, leaving important gap in our understanding of human

142 microbiota in a highly heterogenous disease. Recently initiated ambitious precision nutrition

143 approaches [30-33] cannot be applied on a highly heterogenous disease before deconstructing

144 the disease into multiple strata and tailoring therapies accordingly.

146 In the present study, we postulated that gut microbiota dysbiosis along the AD continuum should

147 reflect an overlapping yet distinct community types. We show that AD gut microbiota includes

148 distinct community types and the cognitive impairments in AD continuum is associated with

149 unique gut microbiota signatures. Elucidating the diversity and community types of gut microbiota

150 would facilitate identification of stratification biomarkers thereby contributing to precision nutrition

151 approaches in AD. 


\section{RESULTS}

\section{Study Design and Participant Characteristics.}

158 The study cohort consisted of $47 \mathrm{AD}, 27 \mathrm{MCl}$ (all amnestic), and 51 subjects non-demented controls $(\mathrm{N}=125)$. To minimize dietary confounding effect on the microbiome, we included healthy co-habiting spouses of the patients sharing the same diet as controls. The control group therefore

161 largely $(n=27)$ comprised partners of the patients. Participants were recruited in two health centers

162 located in different cities. The cohort groups were statistically not different in terms of sex, but age

163 and education factors were significantly different (Table 1), therefore statistically adjusted in 164 analyses. Expectedly, the groups were also different in cognitive tests including the Mini-Mental 165 State Exam (MMSE), and the Clinical Dementia rating (CDR). Most AD participants had very mild 166 or mild dementia, with clinical dementia rating (CDR) scores ranging from 0.5-3 (median CDR 1

167 for $\mathrm{AD} ; 0.5$ for $\mathrm{MCl}$ and 0 for the control group). The median MMSE scores were significantly 168 higher in control (MMSE=27) and $\mathrm{MCl}(\mathrm{MMSE}=26)$ groups than $\mathrm{AD}$ (MMSE=16). A subset of AD 169 patients $(n=12)$ was clinically asked to undergo lumbar puncture to ascertain diagnosis using CSF 170 biomarkers including $A \beta 42 / A \beta 40$ ratio, phosphorylated tau ( $p$-tau), and the $p$-tau/A $\beta 42$ ratio

171 (Supplementary Table S1). We collected medication information from the patient's registry.

Microbiome composition is associated with disease status along the AD continuum

175 The gut microbiota was profiled using the V3-V4 hypervariable region of the 16S rRNA gene; The 176 Nephele automatic pipeline denoised the paired-end sequences and assigned amplicon 177 sequence variants (ASVs) according to DADA2 [34]. The Nephele produced both unrarefied and 178 the rarefied ASV tables. The rarefied table included a total of 3486 ASVs in the table (10769 sequences/sample) for downstream analyses. 
180 The phylum level taxonomic analysis showed typical human gut microbiota profile in terms of

181 over-abundance of Firmicutes, Bacteroidetes, and Proteobacteria (Figure 1a). Together with

182 Verrucomicrobia, and Actinobacteria the five phyla comprised $99 \%$ of all reads but Proteobacteria

183 was overrepresented in $A D$ patient samples. Notably, the genus level relative abundance

184 distributions across samples showed Prevotella_9 and Bacteroides were the most abundant of

185 top30 genera across the samples (Figure 1b). To perform differential abundance analysis

186 between samples we sought concordance analysis among multiple tools. ANCOM-BC or

187 ALDEx2, when used covariates in their models, both agreed that only Ruminoccus_unclassified

188 is significantly differentially abundant among the groups (data not shown). Nevertheless, when

189 we employed limma-voom R package (age and sex adjusted, FDR<0.05) we found that

190 Prevotella_9, Bacteroides and members of Ruminococcaceae family were among the top most

191 significant differentially abundant taxa (ASV) between the cohort groups (Supplementary Tables

192 S2-5 ). A comprehensive comparative statistical assessment of multivariate and compositional

193 methods [35] demonstrated ALDEx2 or alike tools suffer from low power while limma-voom and

194 songbird in their own class were the best performers.

196 Alpha diversity indices (Shannon, Inverse Simpson) did not show significant differences after 197 multiple testing corrections (Kruskal-Wallis, Supplementary Figure S1 (a-d), FDR>0.05) but 198 richness index, Chao1, showed significant difference between $\mathrm{MCl}$ and the control group 199 (pairwise Wilcoxon rank sum test, $p=0.008074$ ).

201 We employed both relative abundances based and recently developed compositionally aware 202 tools, namely DEICODE [36] and Songbird [37] to compare the composition and structure of 203 bacterial communities in samples using multiple beta diversity indices (Bray-Curtis, Jaccard, and 204 Aitchison). The principal coordinates analysis showed separation of the three groups by both 205 Bray-Curtis and Jaccard indices (Figure 2a-b). We used adonis2 function in qiime2 plugin (q2- 
diversity) to perform PERMONAVA analysis with 999 permutations and included interaction terms

207 (Supplementary Table S6) and seperation of the groups were highly significant $(P=0.0001)$. Age

208 and Sex also significantly contributed to the total variance $(P<0.001)$ but the interaction terms

209 were not significant. Furthermore, dispersion between groups tests (PERMDISP) indicated only

210 the dispersion $\mathrm{MCl}$ group is significantly heteregenous (pairwise comparisons $p=0.033$ for AD-

$211 \mathrm{MCl} ; \mathrm{p}=0.024$ for $\mathrm{C}-\mathrm{MCl} ; \mathrm{p}=0.672$ for $\mathrm{AD}-\mathrm{C})$, which may be attributed to unbalanced design. We

212 added further support for the seperation of the three groups from other ordinations. The Canonical

213 Analysis of Principal Coordinates (CAP) analysis unambigiously showed the three groups are

214 distinct (Figure 2c, trace statistic $=0.86855, \mathrm{p}=0.001,999$ permutations). The final support in beta

215 diversity was provided by the DEICODE analysis (robust Aitchison PCA) (Figure 2d,

216 PERMANOVA $\mathrm{p}=0.02$ ), which indicated that the three groups are distinct, and the community

217 clusters are largely driven by a subset of ASVs with taxonomic assignment Prevotella_9,

218 Bacteroides, a unclassified genus within Ruminococcaceae family

219 (Ruminococcaceae_unclassified), and Escherichia/Shigella. Moreover, the co-occurrence

220 analysis using SparCC showed that Prevotella_9 and Bacteroides were negatively correlated

221 (Correlation=-0.4445, FDR =0.09355). Moreover, the genus level PCoAs showed partially

222 overlapping clusters of these two taxa while the groups overall were also significantly separated

223 (PERMANOVA, $\mathrm{p}<0.0001$, Supplementary Figures S2 (a-c)). We therefore placed particular

224 attention to these two taxa in the rest of the downstream analyses.

226 Enrichment analysis by multinomial regression embedded in the songbird tool with regard to 227 covariates (formula: Age+Sex+Edu+MMSE+CDR+Groups(levels=(“C”, "MCl”,"AD”)) indicated 228 that the natural log ratio of Prevotella_9 to Bacteroides and Prevotella_9 to Escherichia/Shigella 229 significantly separated AD group from the control group (Welch's t-test, FDR adjusted $p=0.04$ ) 230 but not from the $\mathrm{MCl}$ group (Figure 3a-d). Importantly, the songbird excluded 25 samples from 231 this analysis due to zero-rich abundances that do not allow for center-log ratio calculations. We 
232 therefore tested the natural log ratio of top $25 \%$ allowing to include all samples in the analysis

233 ("Set1" in Supplementary Table S7) to the bottom 25\% ("Set2", Supplementary Table S8) of the

234 ranked $\mathrm{ASV}$ s associated with the $\mathrm{AD}$ relative to the control group; also, same ratios for $\mathrm{MCl}$

235 relative to the control group ("Set3" and "Set4", Supplementary Table S8 ) and the ASVs

236 enriched in each group were visualized with Qurro [38]. Both sets of ranked log ratios revealed

237 significant differences (Graph Pad Prism) between the log ratios of features differentiating groups

238 (Welch's t-test, FDR adjusted $p=0.0002$ ).

\section{Discrete multiple subsets of gut microbiota exist along the AD continuum}

242 Considering the preceding results, we postulated that gut microbiota profile along the $A D$ 243 continuum does not represent a single state, rather, distinct yet overlapping community types. We 244 addressed this hypothesis using four unique methods: 1- Partitioning around medoid (PAM)245 based clustering [39], 2- Fitting Dirichlet multinomial mixture (DMM) models to partition microbial 246 community profiles into a finite number of clusters [40] using the Laplace approximation, 3- Fitting 247 Latent Dirichlet Allocation (LDA) [41, 42] using perplexity measure, and 4- Analyzing topological 248 futures of data density [43] based on the Mapper algorithm to capture subtle and non-linear 249 patterns of high-dimensional datasets and population level stratification.

251 The PAM-based clustering identified three $(\mathrm{k}=3)$ distinct clusters based on Gap statistics 252 (Supplementary Figure S3a). PCoA analysis of the sample abundances in the three clusters 253 indicated significant separation of the clusters (Figure 4a, PERMANOVA, p=0.001). We 254 confirmed optimum number of clusters using both Jensen-Shannon and Bray-Curtis distance 255 metrices (data not shown). The relative abundance of the genus Prevotella_9 dominated cluster2561 while the genus Bacteroides showed the highest relative abundances in the other two clusters 257 (Figure 4b). 
259 Next, we employed the Dirichlet multinomial mixtures probabilistic community modeling using the

260 DirichletMultinomial R package [40] and fitting genus level absolute abundances. Based on

261 Laplace approximation three clusters (cluster 1, 2, and 3) represented the best model fit

262 (Supplementary Figure 3b), which was congruent with the PAM-based clustering. The PCoA 263 analysis of these clusters and PERMANOVA pairwise tests further supported existence of three 264 distinct clusters within the microbial community (Figure 4c, PERMANOVA, $p=0.01$ ). The genus 265 Bacteroides was the most abundant taxa in the first two clusters and the third cluster was 266 dominated by Prevotella_9 (Figure 4d). Notably, cluster2 included significantly higher abundance 267 of Bacteroides (26.3\%) than cluster1 (9.9\%) and cluster3 (4.7\%). In addition to highly enriched 268 Bacteroides in cluster2 the decreasing trend of Faecalibacterium abundance and elevated 269 abundance of inflammation associated Escherchia/Shigella suggested that cluster2 can be 270 named "Bacteroides2 (Bact2) enterotype" as recently described [44, 45]. Reportedly, abundance 271 of Bacteroides in Bact2 enterotype can reach as high as $78 \%$ in patients with inflammatory bowel 272 disease and is associated with systemic inflammation. These results suggest that cluster2 273 includes patients with aggravated systemic inflammation.

275 We also performed SIMPER analysis based on Bray-Curtis distance to identify taxa contributing 276 most to dissimilarities between clusters (data not shown). Bacteroides, Prevotella_9, 277 Faecalibacterium, and taxa within Ruminococcaceae family ranked among the top taxa 278 contributing most to differences between the three DMM clusters. To examine which factors were 279 associated with the DMM clusters we analyzed distribution of clinical metadata and diversity 280 metrics within the clusters. Alpha diversity indices (Chao1, Shannon, and Inverse Simpson) were 281 statistically different between all three clusters after Benjamini-Hochberg FDR adjustment. 282 However, CDR, MMSE, Age, Sex, and Education were not significant between the clusters 
283 (Kruskal Wallis test followed by Dunn's posthoc test, FDR $<0.05$ and Fisher's Exact test was used

284 for Sex parameter). (Figure 5 a-h)

286 We next tested LDA potential to stratify gut microbiota of the cohort participants. This 287 unsupervised machine learning technique is increasingly finding acceptance in the field of 288 microbiome [46-48] for its unique ability to reveal latent or hidden groups within the data cloud.

289 Supplementary Figure S4 shows LDA model's perplexity parameter and log-likelihood values to 290 find optimal number of clusters. Both parameters continued to partition the community without 291 reaching a clear optimum. This finding is unexpectedly consistent with recent publications using 292 LDA in microbial ecology [46-48]. Bacteria probability distributions (ranked by probability $\geq 1 \%$ in 293 descending order) across the subgroups are displayed in Figure 6a. Interstingly, of the ten 294 subgroups two subgroups were dominated by Bacteroides (topic1 and topic5) and a subgroup 295 (topic2) dominated by Prevotella_9 with 97\% probability. These subgroups therefore resemble subgroups detected by PAM and DMM in terms of prevalence of Bacteroides and Prevotella_9.

297 Unlike DMM and PAM, however, LDA detected a distinct subgroup (topic10) with top ranking 298 genus was Escherichia/Shigella, which also included putatively opportunistic bacteria such as

299 Entercoccus and Klebsiella. Subgroups 4, 6, and 9 were conspicious with the genera known to 300 produce butyrate and acetate or is mucinphilic. Even though we present first ten subgroups 301 (topics) here we also examined higher order subgroups and observe that the ten subgroups are 302 further partitioned into additional subgroups such as subgroups with topranking probability of 303 Lactobacillus and Akkermansia emerge. Finally, we plotted Quetelet index by subgroups to infer 304 associations between subgroups and the cohort groups (Figure 6b). Quetelet index estimates the 305 relative change of the occurence frequency of a latent subgroup among all the samples compared 306 to that among the samples of the cohort groups. The index showed subgroups $1,8,9,10$ are 307 positively associated with $A D$ group. The subgroup 9 is enriched by the members of 308 Ruminococcaceae family. The top ranking Ruminococcaceae_UCG_002 and Akkermansia are 
309 more abundant in AD group than the control group according to limma-voom analysis.

310 Akkermansia overabundance in AD gut microbiota is counterintutive but was previously reported

311 by others [25] and this genus is more abundant in the gut microbiota of Parkinson's patients, also

312 [49]. The subgroup 10, where Escherichia/Shigella is the top ranking genus, is strongly associated

313 with AD group but negatively associated with other groups. Conversely, subgroups 2,4, and 7,

314 which are enriched by short chain fatty acid producers, are positively associated with the control

315 and $\mathrm{MCl}$ groups but negatively associated with $\mathrm{AD}$.

317 Another and last method we employed to stratify gut microbiota was topological data analysis

318 (TDA), based on the Mapper algorithm [50] embedded in recently developed tmap tool [43]. The

319 tmap tool was developed for network representation for stratification and association study of

320 high-dimensional microbiome data. After constructing TDA microbiome network using Mapper

321 algorithm (ordination, covering, and DBSCAN clustering) the workflow in the second step includes

322 computation of a modified version of the spatial analysis of functional enrichment (SAFE) scores

323 to map both the metadata and microbiome features into the TDA network to generate their vectors

324 of SAFE scores. Vectors of SAFE scores are then used to perform ranking and ordination, and

325 co-enrichment relations to delineate relationship between metadata and microbiome features. To

326 construct TDA network we first applied dimension reduction (filtering) in PCoA using Bray-Curtis

327 distance, followed the above algorithm and also repeated the entire analysis using Jensen-

328 Shannon distance to reveal effect of distance metric, if any. To understand how driver taxa relate

329 to each other and with the clinical metadata we performed Principal Component Analysis (PCA)

330 of SAFE scores. Figure (7a) shows the TDA network and PCA (Bray-Curtis distance) of taxa-

331 metadata based on SAFE scores (Supplementary Table S9), respectively. We obtained similar

332 TDA network profile using Jensen-Shannon distance (Figures 7b) and SAFE scores

333 Supplementary Table S10). Size of each marker is scaled according to the SAFE score and only

334 top30 bacteria species are shown in PCA figures for clarity. A node in the network represents a 
335 group of samples sharing similar bacteria genus profiles. Two given nodes are linked when

336 common samples are shared between the two nodes. The TDA analysis using both distance

337 indices resulted in very similar stratification profile with the top ten SAFE scoring genera included

338 Prevotella_9, Bacteroides, Rumunococaceae_unclassified, species of Lachnospiraceae, and

339 GCA90006675. Unsurprisingly, a few taxa ranking differed between the two profiles such as

340 Caprococcus_2, Mollicutes_RF39_unclassified.

342 Furthermore, Figures (8a and $8 b$ ) show taxa and host covariates based on Bray-Curtis and

343 Jensen-Shannon distances, respectively. Regardless of the distance metric, all three groups were

344 clearly separated. The drivers of microbiome stratification (Prevotella_9, Bacteroides,

345 Ruminococcus_unclassified) are placed near the control, $\mathrm{AD}$ and $\mathrm{MCl}$ groups, respectively in both

346 PCA figures. Of the clinical metadata, MMSE, sex, and education were grouped with the control

347 group and co-enriched with Prevotella_9 but also with Prevotella_2, and Haemophilus, and

348 Lachnospiraceae_NK4B4_group. Conversely, CDR, age, and AD group were clustered together

349 and co-enriched with taxa such as Subdoligranulum, Odoribacter, Bilophila, Alistipes. The MCl

350 group was co-enriched with Ruminocoaceae_unclassified, Mollicutes_RF39_unclassified,

351 Ruminocoaceae_UCG_005, Lachnospiraceae_unclassified. However, some taxa such as

352 Odoribacter was placed near the control group in Jensen-Shannon distance PCA Figure (8b),

353 suggesting co-enrichment of certain taxa can be somewhat influenced by the preferred distance

354 metric.

355 Identification of signature taxa for AD continuum and association with metadata

357 We constructed Random Forest (RF) model on selected features of gut microbiota and 358 psychometric test scores (MMSE and CDR) that are typically used as proxy in clinical diagnosis.

359 Using songbird, we selected 300 ASV (Top 25\%) that differentiates between the healthy (control)

360 and the disease groups ( $\mathrm{MCl}$ and $\mathrm{AD})$. We then plotted the ASVs with the first 20 highest mean 
361 decrease Gini values (Figure 9a) and included ASVs with mean decrease Gini values above the

362 breakpoint curve in the RF analysis. We identified the following 9 ASVs above the breakpoint:

363 Faecalibacterium (ASV45), Sutterella(ASV607), Coprobacter(ASV531), Bacteroides (ASV81),

364 Anaerostipes(ASV364), Ruminoccocaceae_unclassified(ASV203), Lactobacillus (ASV65),

365 Clostridium_sensu_stricto_1 (ASV118), Ruminococcus_1 (ASV59). Notably, ASVs beyond the

366 breakpoint line are largely the bacterial species responsible for the stratification of gut microbiota

367 in the samples such as Faecalibacterium, Bacteroides, and Ruminococcus_unclassified. We next

368 calculated diagnostic accuracy of the RF model by plotting receiver operating characteristics

369 curve (ROC) for the above 9 taxa, MMSE, and CDR separately and in combination for each cohort

370 group (Figure 9b). The ROC value for these selected nine taxa were moderately accurate (AUC

$37163 \%$, Flg 8a) but when we included MMSE and/or CDR, we found that the RF model robustly

372 classify all three groups (groupwise AUC range 0.74-1.0, Figures 9b).

374 Taxa association with clinical parameters

376 We used multivariate association with linear models (MaAsLin2) to assess association between 377 individual taxa and clinical metadata including patients drugs ( $q \leq 0.25)$. This analysis showed 378 that Roseburia, Lactobacillus, Fusicatenibacter were negatively associated with AD 379 (Supplementary Figure S5). Of the medication categories there are several taxa found to be 380 positively associated with anti-depression and statin. Blautia, Caprococcus, Butyricoccus, Dorea, 381 Lachnospiraceae family members, some Ruminoclostridium and Ruminococaceae, known to be 382 butyrate producers are all positively associated with antidepression drugs. Unexpectedly, we 383 found that several taxa were significantly associated with Statin medication and, of these taxa, 384 Streptococcus and unclassified member of Erysipelotrichaceae were highly significantly 385 associated with statin medication. We also observed the following taxa positively associated with 386 statin medication; unclassified members of Ruminococaceae and Lachnospiraceae, 
Phascolarctobacterium, Desulfovibrio, Caprobacter, Bifidobacterium, Butyricoccus, Blautia, Barnesiella.

\section{DISCUSSION}

391 In this study, we demonstrate that gut microbiota across AD continuum not only differentiates 392 between cognitive states but also comprise subgroups delineated by locally dominant co393 occurring bacteria. Stratification of the gut microbiota along the AD continuum is major unmet 394 need for diet-based and precision nutrition interventions in AD cohorts and here we present proof395 of-concept data that can be insighful for the emerging dietary and precision medicine/nutrition 396 initiatives involving AD patients. A key finding in this study is that these approaches all converge 397 on Prevotella and Bacteroides stratification, which are also robustly supported by enrichment and 398 ordination analyses that these two species are the drivers of community diversity and 399 composition. Rather than focusing on a single gut microbiota stratification method we have 400 exercised the best practice of implementing multiple methods to compare, contrast, and sought 401 support from alternative analyses. Also, all methods ranked the following taxa among the Top10 402 bacteria contributing to seperation of the groups; Escherchia/Shigella, Faecalibacterium, Blautia, 403 Ruminococcaceae_unclassified, Ruminococcaceae_UCG-002, Lachnospiraceae_unclassified, 404 Parabacteroides, suggesting these taxa play significant role in the observed community structure 405 of the gut microbiota of the patients in this study.

407 PAM clustering and DMM concordantly showed three distinct clusters, one of which is consistent 408 with the recently described Bact2 group [44]. The subjects in this group are likely to have 409 aggravated dysbiosis as manifested from increased abundance of opportunistic pathogens 410 Escherichia/Shigella and some species of Bacteroides species and lower abundance of 411 Faecalibacterium and other SCFA producers. Notably, LDA analysis shuffles similar set of taxa 
412 as the number of subgroups increase but Bacteroides and Prevotella_9 are predominantly the

413 most abundant taxa in many of these clusters. Strikingly, Escherichia/Shigella dominates one of

414 the subgroups in LDA analysis together with opportunistic Klebsiella and Enterococcus,

415 suggesting dysbiotic community type may be enriched in this subgroup.

417 Topological data analysis (TDA) we used to stratify gut microbiota in this study deserves a 418 particular attention among others. TDA, based on the Mapper algorithm [50], represents the 419 underlying distribution of data in a metric space by dividing the data into overlapping similar 420 subsets according to a filter function, local clustering on each subset and representing the results 421 in an undirected network. A node in the network represents a group of samples with similar 422 microbiome profiles, and if common samples between nodes are shared then the nodes are 423 linked. Next, a modified special analysis of functional enrichment (SAFE) algorithm maps the 424 metadata and taxa into the network. Finally, vectors of SAFE scores can be used in ordination to 425 rank the driver taxa and their relationship with the metadata, all these algorithms are integrated 426 into tmap [43]. The SAFE scores we obtain following these algorithms allowed us to identify the 427 driver species that are responsible for community structure and showed their relationship with the 428 metadata. We employed Bray-Curtis and Jensen-Shannon to check the variation resulting from 429 distance metric. Prevotella_9, Bacteroides, and Ruminoccus_unclassified were ranked among 430 the top10 taxa with high SAFE scores, albeit in different order, suggesting TDA is robust and 431 consistent even with different distance metrics. In addition to these three taxa unclassified 432 members of again other taxa within Ruminoccus family and Lachnospiraceae were congruent 433 with other three methods we tested. Interestingly, this analysis identified GCA-900066575 taxa 434 (Uncultured human intestinal bacterium) as one of the subclusters in contrast with other methods 435 we used. This genus is taxonomically in the family of Lachnospiraceae, which includes members 436 of SCFA producers [51], still some other members were associated with metabolic diseases such 437 as obesity [52]. Indeed, another related member of this family GCA-900066225 ranked among 
438 the top10 taxa when Bray-Curtis distance was used but enriched around AD. It is therefore

439 important to note that TDA, unlike clustering or probabilistic partitioning methods, provided fine

440 resolution in terms of stratification of the gut microbiota composition. Conversely, TDA did not

441 rank Escherchia/Shigella subnetwork among top ten taxa, neither the ordination showed clear

442 association with the disease. Together, bioinformatic tools developed in the field of microbiome

443 have all their strengths and drawbacks and therefore overlaps in bioinformatic analyses should

444 be pursued.

446 Several lines of evidence showed human cohorts in microbiome studies can be phenotypically

447 partitioned along Prevotella and Bacteroides stratification [53-58]. A recent comprehensive report

448 [59] provided evidence that Mediterranean diet-based intervention is associated with specific

449 functional and taxonomic components of the gut microbiome, and its effect is a function of

450 microbial composition. Notably, absence of Prevotella copri in the gut microbiomes of a subgroup

451 of participants was associated with the protective health benefits of the dietary intervention,

452 emphasizing the premise that microbiome-informed stratified dietary intervention would be quite

453 effective. Nevertheless, $P$. copri is ambivalently associated with both heath and diseases

454 depending on the strain and geography [60], which prompts us to further consider its role in AD.

456 Taxonomically, the genus Prevotella_9 is predicted to belong to Prevotella copri complex [61].

457 Comparative genome analysis of the strains of $P$.copri complex, however, show that some strains

458 qualify to be assigned to even a separate species of Prevotella due to low genomic similarities

459 [62,63]. Some $P$. copri strains are associated with disease states such as rheumatoid arthritis

460 [64], while some other strains are associated with habitual diet and life style [54] and 461 underrepresented in Westernized populations. Thus, strain level resolution of Prevotella_9 is 462 needed to draw inferences. Expectedly, multiple strains of $P$. copri are likely to be part of the 463 bacterial community in the samples. Even though we found Prevotella_9 to be associated with 
464 the control group the enrichment analysis using songbird ranked some ASVs belong to

465 Prevotella_9 (species level) at the top and few other ASVs at the bottom of the log ratio

466 differentials, suggesting analysis beyond species taxonomic hierarchy would provide better

467 resolution in terms of their associations with human phenotypes. Oligotypes of these two genera

468 in an earlier work were found to be differentially associated with plant based or some others were

469 associated with animal-based diet [55]. A recent report provided evidence that Bacteroides

470 cellulosilyticus predicted weight gain more precisely than the ratio of Prevotella and Bacteroides

471 genus. Together, our differential enrichment analysis results are in line with these reports that

472 species or even strain level resolution of these two genera could provide better predictive

473 biomarker power for diet-based intervention studies.

475 One limitation of our study was that although we were able control drug induced confounding, we

476 did not control other potential confounders such as diet, BMI, stool consistency. We largely

477 recruited cohabiting spouses as non-demented controls sharing the same diet patterns with the

478 patients and carnivory is rare due to the high cost of meat in the country. We therefore did not

479 predict diet can strongly impact our results.

481 In conclusion, we demonstrate in this study that gut microbiota along the Alzheimer's Disease

482 continuum comprises stratified community structure dashed primarily by Prevotella and

483 Bacteroides but also subnetworks of other taxa exist in the community. The signature taxa when

484 used together with MMSE and CDR robustly classify heterogenous groups hence posing potential

485 biomarker value. The study adds to limited number of clinical studies profiling gut microbiota of

486 AD continuum patients. 


\section{MATERIALS AND METHODS}

492 Subject Recruitment and Study Design: The Istanbul Medipol University and Erciyes University 493 Ethical Review Boards approved this study (Approval numbers: 186/16.4.2015 and 85/ 49420.02 .2015 , respectively). All participants were informed of the objectives of this study and signed 495 a written consent form prior to their participation. The diagnosis of dementia and $\mathrm{MCl}$ due to $\mathrm{AD}$ 496 were based on the criteria of the National Institute on Aging-Alzheimer's Association workgroups 497 on diagnostic guidelines for Alzheimer's disease $[65,66]$. Exclusion criteria for this study included 498 history of substance abuse, any significant neurologic disease, major psychiatric disorders 499 including major depression. Also, individuals who used commercial probiotics or antibiotics during 500 the study period or within 1-month prior to providing stool sample, or who major GI tract surgery 501 in past 5 years. Both health centers followed the same protocols in recruiting cohorts and used 502 kits from the same manufacturers to minimize the variations in wet lab procedures.

Lumbar puncture, CSF biomarkers assays: Cerebro Spinal Fluid (CSF) samples were included

505 in the analyses from a subset of AD patients if the patient was requested to donate CSF sample 506 as part of the clinically mendated diagnostic protocol. CSF samples were collected in the morning 507 after overnight fasting using spinal needles (22 gauge) and syringes at the L3/4 or L4/5 interspace.

508 CSF was then aliquoted into $0.5 \mathrm{~mL}$ non-adsorbing polypropylene tubes and stored at $-80{ }^{\circ} \mathrm{C}$ 509 until assay. Biomarker molecules in CSF $\left(A \beta_{42}\right.$, phosphorylated tau ( $p$-tau), and the $p$-tau/A $\beta 42$ 510 ratio) were measured consistent with the Alzheimer's Association flowchart for lumbar puncture 511 and CSF sample processing and the biomarker levels were determined as previously described 512 [67]. Single 96-well ELISA kits (Innogenetics, Ghent, Belgium) were used in quantitation.

514 Sample collection and DNA extraction: Stool samples from all participants were collected in 515 the neurology clinics of the university training hospitals. The participants were given a collection 
516 kit included a sterile tube and provided a brief instruction for collection. Self-collected samples

517 were placed within approximately 30 mins of collection in -80 freezers and kept frozen until DNA

518 extraction.

16S rRNA gene sequencing and PCR were performed as previously described [68] with minor

521 modifications. Briefly, genomic DNA was extracted from $220 \mathrm{mg}$ fecal samples using QiaAmp

522 DNA Stool Mini Kit (Qiagen, Germany) per manufacturer's instructions with the addition of bead

523 beating ( $0.1 \mathrm{~mm}$ zirconium-beads) and lysozyme and RNAse A incubation steps.

PCR and amplicon sequencing: To amplify the variable V3-V4 regions of the 16S rRNA gene,

526 the primers $341 \quad \mathrm{~F} \quad\left(5^{\prime}-\mathrm{CCTACGGGNGGCWGCAG-3}\right)$ and $805 \quad \mathrm{R} \quad\left(5^{\prime}-\right.$

GACTACHVGGGTATCTAATCC-3') were used. MiSeq sequencing adaptor sequences were

531 following conditions were followed: denaturation at $95{ }^{\circ} \mathrm{C}$ for 3 min., followed by 25 cycles of

532 denaturation at $95{ }^{\circ} \mathrm{C}$ for $30 \mathrm{sec}$., annealing at $55^{\circ} \mathrm{C}$ for $30 \mathrm{sec}$. and extension at $72{ }^{\circ} \mathrm{C}$ for 30

533 sec., with a final extension at $72{ }^{\circ} \mathrm{C}$ for $5 \mathrm{~min}$. Amplified PCR products were purified with

534 Agencourt AMPure XP purification system (Beckman Coulter) and Nextera PCR was performed

535 by using sample-specific barcodes. The constructed Nextera libraries were then sequenced by

536 Illumina MiSeq platform using MiSeq Reagent Kit v2 chemistry.

538 Sequence processing and taxonomic assignment: The pair-end 16S rRNA reads were first

539 used cutadapt v1.9 program [69] for the process of quality filtering, trimming and uploaded on the

540 DADA2 pipeline [34] integrated into the Nephele platform [70] (v.2.0, http://nephele.niaid.nih.gov).

541 Chimeric sequences are automatically removed by this pipeline, which generates both rarefied 
542 and unrarefied ASV abundance tables. We used Rarefied (10769 reads/sample) ASV table in

543 most downstream analysis due to large differences between some total sample reads except for

544 the scale invariant DEICODE and songbird. We removed any sequences that were classified as

545 either being originated from eukarya, archaea, mitochondria, chloroplasts or unknown kingdoms.

547 Quality control: We included no sample DNA extractions and no template negative control 548 samples in every sequencing library prepared. Using reads in the negative control samples as 549 reference we identified and removed probable contaminant reads of 13 ASVs from the ASV table, 550 as predicted by Decontam R package [71] using the 'prevalence' method. In this method, the 551 binary coded features across samples are compared to the prevalence in negative controls to 552 identify contaminants. Also, we sequenced the same amplicon of an AD sample three times to 553 check the sequencing variation. Although both centers used same protocols and kits from the 554 same manufacturer in sequencing, we sequenced amplicons amplified from two same genomic 555 DNA templates again from AD samples at both centers to check the center-to-center sequencing 556 concordance. No differences could be identified between the taxonomic compositions of the 557 samples seuquenced at both centers nor between the technical replicates (PCoA, PERMANOVA $558 p=0.1)$

Numerical Ecology and Statistical Analysis: Most numerical downstream analysis of ASV

561 abundances were performed in $\mathrm{R}$ environment [72]. All $\mathrm{P}$ values, where appropriate, were 562 adjusted for multiple testing using Benjamini-Hochberg (False Discovery Rate; FDR) method. We 563 measured within samples microbial diversity (alpha diversity) using Observed richness, Chao1, 564 Shannon, and Inverse Simpson in phyloseq [73] and tested using Kruskal Wallis. To identify 565 differentially abundant bacterial species we employed animalculus [58] and limma [74] $R$ 566 packages. We assessed microbial diversity between samples (beta diversity) using multiple 567 distance metrics including Bray-Curtis, Jaccard, Canonical Analysis of Principal Components 
568 (CAP). CAP analysis and the similarity percentages breakdown (SIMPER) procedure were 569 performed using PRIMER.v7 [75]. Additionally, due to the compositional nature of the data, we 570 also included robust Aitchison PCA, using the Qiime2 DEICODE plugin [36] to calculate beta 571 diversity with feature loadings. The resulting ordination was visualized using Emperor [76]. We 572 tested significance of beta diversity among groups using again Qiime diversity plugin 573 PERMANOVA.

575 Next, we used Songbird [37] for multinomial regression to rank species association with disease 576 status with the following parameters: (formula: "MMSE+CDR+Sex+Edu+C(Group, Diff, 577 levels=('C','MCl','AD'), -p-epochs 10000 --p-differential-prior 0.5 --p-summary-interval 1 --p578 random-seed 3 -min-sample-count 1000 -min-feature-count 0 ). Of note, the formula structure 579 follows Patsy formatting (https://patsy.readthedocs.io/en/latest/ ) such that Groups (C, MCI, AD) 580 represent levels=["healthy", "mild", "severe"] states, respectively. A null model was generated 581 using the same parameters. The fitted model demonstrated better fit compared to the null model 582 (pseudo $\mathrm{Q}^{2}=0.874027$ ). Taxa ranks were visualized using Qurro [38]. Significance was 583 determined using a Welch's t-test between groups, performed by Graph Pad Prism.

585 To identify microbial species associated with the clinical metadata including patients' medication 586 we performed multivariate association with linear models (MaAsLin2) [77]. The control group was 587 excluded from this analysis as they were not normally prescribed these medications. We 588 employed the R package MaAsLin 2.1.0 to perform per-feature tests. We log-transformed relative 589 abundances of microbial species and standardized continuous variables into Z-scores and binary 590 encoded medication information before including them in the MaAsLin models $(q<0.25$ for 591 significance). 
593 Stratification of gut microbiota: We employed clustering, probabilistic partitioning, and

594 topological data analysis approaches for the stratification of gut microbiota in the samples.

595 Partitioning around the medoid (PAM) approach [39] clusters samples by iteratively updating each

596 cluster's medoid. We assigned samples to community types using the function pam() in $\mathrm{R}$

597 package cluster based on Bray Curtis and Jensen Shannon distances. The number of clusters

598 was determined by Gap statistic evaluation. Departing from the clustering approach, we next used

599 two distinct probabilistic methods to partition microbiota landscape, namely Dirichlet multinomial

600 mixture models (DMM) [40] and Latent Dirichlet Allocation (LDA) [41, 42]. Genus level

601 abundances were fitted to DMM models to partition microbial community profiles into a finite

602 number of clusters, using the Laplace approximation as previously described $[40,78]$.

604 As a second probabilistic partitioning we performed LDA, is a multi-level hierarchical Bayesian

605 model [41] otherwise used for collections of discrete data such as text corpus analysis in

606 linguistics. LDA is a generalization of Dirichlet multinomial mixture modeling where biological

607 samples are allowed to have fractional membership and distinct microbial communities have

608 different microbial signatures. Thus, for each taxon there is a vector of probabilities across all

609 clusters that it can be assigned to. Each cluster, therefore, has a different probability of containing

610 taxa, indicating chance of microbes in a particular subgroup (strata) co-occurring due to

611 community assembly dynamics. To fit the model we used Gibb's sampling with the R package

612 MetaTopics (v.1.0) [79]. The relative abundances of genus collapsed table with abundances more

613 than $0.1 \%$ and $5 \%$ sample prevalence was input to the model. We plotted perplexity measure and

614 loglikelyhood values to estimate model performance and optimal number of topics (subgroups of

615 microbial assemblages) using 5-fold cross-validation. However, we observed that both

616 parameters continued to improve with increasing subgroup number without a clear optimum

617 except the first jump in perplexity was near 10 topics. We therefore picked first 10 topics for the 618 sake of interpretability. 
620 The final method we applied was topological data analysis (TDA) based on the Mapper algorithm

621 [50] and network representation for stratification and association of study of high dimensional 622 microbiome data, all integrated into tmap tool [43]. The framework enables to reveal association

623 of taxa or metadata within the entire network and to identify enrichment subnetworks of different

624 association patterns. Conceptually, the Mapper algorithm transforms a distance matrix and

625 represent the shape of the data cloud in an undirected network. Next, a modified version of special

626 analysis functional enrichment (SAFE) algorithm to map the value of the target feature into the

627 network was employed, followed by ordination of SAFE scores to show taxa-metadata association 628 [43].

Signature taxa: To identify microbial signature of severity of cognitive impairment in $A D$

631 continuum we implemented a machine learning procedure. We first took advantage of songbird

632 tool to select features including the covariates and healthy (control) and disease states (AD+MCI)

633 in the model formula. We subsequently fit the list of ASV selected this way into Random Forest

634 models. We plotted the area under the receiver operating characteristic curve (AUROC) to show

635 prediction performance of the models. To create the classifiers, a random forest constituted of

636500 trees were computed using the default settings of the "randomForest" function implemented

637 in the randomForest R package (v4.6-7). Mean decrease Gini values were averaged for each

638 ASV among the 100 random forest replicates. The ASVs with the first 20 highest mean decrease

639 Gini values were plotted. ASVs with mean decrease Gini values above the breakpoint curve were

640 chosen to be part of the classifier. Breakpoints were estimated using the "breakpoints" function

641 included in the strucchange R package [52]. We subsequently fit the list of ASVs selected this

642 way with or without psychometric test values, i.e. MMSE and CDR, into Random Forest models,

643 and bootstrapped for 100 times. We plotted the area under the receiver operating characteristic

644 curve (AUROC) to show prediction performance of the models. 


\section{DATA ACCESSION}

648 The 16S rRNA generated by this study have been submitted to the NCBI BioProject database,

649 (https://www.ncbi.nlm.nih.gov/bioproject/) under accession number PRJNA734525.

\section{AUTHOR CONTRIBUTIONS}

Conception and Design: SY, OUN, EK, and LH; Sample Collection and Processing: BS, AG,

654 FK, MFG, DK, AES, HAV, EAG, and KS; Data Analysis: SY, OUN, AB, MA, and MK; Data

655 Interpretation: SY, OUN, MK, AM, LH and EK. Manuscript Writing - Original Draft: SY; Writing, 656 Review, and Editing: OUN, MK, AM, LH and EK. All authors read and approved the final 657 manuscript.

658

\section{DISCLOSURE DECLARATION}

660

661 The authors do not have any conflicts of interest to disclose.

662

\section{LEGENDS FOR SUPPLEMENTAL TABLES AND FIGURES}

665 Table S1. Levels of Cerebro-Spinal Fluid Biomarkers of a Subset of AD Patients.

666 Table S2-S5. Differentially Abundant ASV and genus level taxa between cohort groups as 667 detected by Limma-Voom Model (Age and Sex Adjusted)

668 Table S6. PERMANOVA analysis of covariates

669 Table S7-S8. Enrichment analysis by multinomial regression embedded in the songbird (Set1, 670 Set2, Set3, and Set4) 
671 Table S9-S10: Ranking of SAFE scores calculated using tmap algorithm

674 Figure S1. Alpha diversity analysis. Box plots show (A) Chao1 index, (B) Inverse Simpson, (C)

675 Observed species, (D) Shannon diversity index

676 Figure S2. Multi-Dimensional Scale (MDS) Analysis of genus relative abundances. (A) MDS

677 analysis of the samples (B) Gradient of Prevotella_9 abundances across the samples. (C)

678 Gradient of Bacteroides abundances across the samples

679 Figure S3. Determining the number of clusters in the gut microbiota. The optimal number of

680 clusters based on (A) Gap statistic with standart error bars for PAM analysis. (B) Laplace method

681 for evaluating model fit for increasing number of Dirichlet mixture components

682 Figure S4. Latent Dirichlet Allocation Model Performance. LDA model's perplexity parameter

683 (top) and log-likelihood values (bottom) to find optimal number of clusters.

684 Figure S5. Associations of the patient drugs with genus-level features. The heatmap shows

685 per-feature testing in MaAsLin 2 using linear mixed models to identify microbial species

686 associated with drugs used by the patients. Colors of the heatmap reflects the beta coefficient for

687 drugs and age and sex from linear mixed models in MaAsLin 2 with genus-level feature as

688 outcomes.

\section{ACKNOWLEDGEMENTS}

691 This study was supported by funding from The Scientific and Technological Research Council of

692 Turkey (TÜBITAK) to Prof. Dr. Süleyman Yildirim and Prof. Dr. Emel Köseoglu (Project numbers.

693 2236-115C056 and 215S707, respectively). The funding agency had no role in study design, data

694 collection and interpretation, or the decision to submit the work for publication. 


\section{References}

698

$700 \quad$ 1. International., A.s.D., Alzheimer's Disease International. World Alzheimer Report 2015:

701 the Global Impact of Dementia. An Analyses of Prevalence, incidence, Cost and Trends.

702 https://www.alz.co.uk/research/WorldAlzheimerReport2015.pdf, Accessed Sep 20, 2020.

2015.

$704 \quad 2 . \quad$ Dubois, B., et al., Preclinical Alzheimer's disease: Definition, natural history, and

705 diagnostic criteria. Alzheimers Dement, 2016. 12(3): p. 292-323.

706 3. Aisen, P.S., et al., On the path to 2025: understanding the Alzheimer's disease continuum. Alzheimers Res Ther, 2017. 9(1): p. 60.

708 4. Kumar, A., A. Singh, and Ekavali, A review on Alzheimer's disease pathophysiology and 709 its management: an update. Pharmacol Rep, 2015. 67(2): p. 195-203.

710 5. Mehta, D., et al., Why do trials for Alzheimer's disease drugs keep failing? A

711 discontinued drug perspective for 2010-2015. Expert Opin Investig Drugs, 2017. 26(6):

712 p. $735-739$.

713 6. Kametani, F. and M. Hasegawa, Reconsideration of Amyloid Hypothesis and Tau

714 Hypothesis in Alzheimer's Disease. Front Neurosci, 2018. 12: p. 25.

$7157 . \quad$ Wang, J., et al., A systemic view of Alzheimer disease - insights from amyloid-beta 716 metabolism beyond the brain. Nat Rev Neurol, 2017. 13(10): p. 612-623.

717 8. Itzhaki, R.F., et al., Microbes and Alzheimer's Disease. J Alzheimers Dis, 2016. 51(4): p. $718 \quad 979-84$

719 9. Guillot-Sestier, M.V., K.R. Doty, and T. Town, Innate Immunity Fights Alzheimer's 720 Disease. Trends Neurosci, 2015. 38(11): p. 674-681. 
721 10. Rangasamy, S.B., et al., Selective disruption of TLR2-MyD88 interaction inhibits inflammation and attenuates Alzheimer's pathology. J Clin Invest, 2018. 128(10): p.

11. Association, A.s., 2020 Alzheimer's disease facts and figures. Alzheimers Dement, 2020.

725 12. Bullain, S. and R. Doody, What works and what does not work in Alzheimer's disease? From interventions on risk factors to anti-amyloid trials. J Neurochem, 2020.

727 13. Bredesen, D.E., Reversal of cognitive decline: a novel therapeutic program. Aging (Albany NY), 2014. 6(9): p. 707-17.

729 14. Bredesen, D.E., et al., Reversal of cognitive decline in Alzheimer's disease. Aging (Albany NY), 2016. 8(6): p. 1250-8.

731 15. Isaacson, R.S., et al., Individualized clinical management of patients at risk for Alzheimer's dementia. Alzheimers Dement, 2019. 15(12): p. 1588-1602.

733 16. Keine, D., et al., Development, Application, and Results from a Precision-medicine Platform that Personalizes Multi-modal Treatment Plans for Mild Alzheimer's Disease and At-risk Individuals. Curr Aging Sci, 2018. 11(3): p. 173-181.

736 17. Fan, Y. and O. Pedersen, Gut microbiota in human metabolic health and disease. Nat $737 \quad$ Rev Microbiol, 2020.

738 18. Fung, T.C., C.A. Olson, and E.Y. Hsiao, Interactions between the microbiota, immune and nervous systems in health and disease. Nat Neurosci, 2017. 20(2): p. 145-155.

740 19. Fang, P., et al., The Microbiome as a Modifier of Neurodegenerative Disease Risk. Cell Host Microbe, 2020. 28(2): p. 201-222.

742 20. Haran, J.P., et al., Alzheimer's Disease Microbiome Is Associated with Dysregulation of 743 the Anti-Inflammatory P-Glycoprotein Pathway. mBio, 2019. 10(3).

744 21. Li, B., et al., Mild cognitive impairment has similar alterations as Alzheimer's disease in 745 gut microbiota. Alzheimers Dement, 2019. 15(10): p. 1357-1366. 
22. Liu, P., et al., Altered microbiomes distinguish Alzheimer's disease from amnestic mild cognitive impairment and health in a Chinese cohort. Brain Behav Immun, 2019. 80: p.

23. Nagpal, R., et al., Modified Mediterranean-ketogenic diet modulates gut microbiome and short-chain fatty acids in association with Alzheimer's disease markers in subjects with

24. Saji, N., et al., Analysis of the relationship between the gut microbiome and dementia: a cross-sectional study conducted in Japan. Sci Rep, 2019. 9(1): p. 1008.

25. Vogt, N.M., et al., Gut microbiome alterations in Alzheimer's disease. Sci Rep, 2017. 7(1): p. 13537.

26. Vogt, N.M., et al., The gut microbiota-derived metabolite trimethylamine N-oxide is elevated in Alzheimer's disease. Alzheimers Res Ther, 2018. 10(1): p. 124.

27. Zhuang, Z.Q., et al., Gut Microbiota is Altered in Patients with Alzheimer's Disease. J Alzheimers Dis, 2018. 63(4): p. 1337-1346.

760 28. MahmoudianDehkordi, S., et al., Altered bile acid profile associates with cognitive impairment in Alzheimer's disease-An emerging role for gut microbiome. Alzheimers

763 29. Amini, Y., et al., The Role of Nutrition in Individualized Alzheimer's Risk Reduction. Curr

30. Isaacson, R.S., et al., The clinical practice of risk reduction for Alzheimer's disease: $A$ personalized nutrition. Nat Rev Microbiol, 2019. 17(12): p. 742-753. 
771 33. Schelke, M.W., et al., Nutritional interventions for Alzheimer's prevention: a clinical precision medicine approach. Ann N Y Acad Sci, 2016. 1367(1): p. 50-6.

34. Callahan, B.J., et al., DADA2: High-resolution sample inference from Illumina amplicon

35. Calgaro, M., et al., Assessment of statistical methods from single cell, bulk RNA-seq,

36. and metagenomics applied to microbiome data. Genome Biol, 2020. 21(1): p. 191. Perturbations. mSystems, 2019. 4(1).

37. Morton, J.T., et al., Establishing microbial composition measurement standards with reference frames. Nat Commun, 2019. 10(1): p. 2719.

38. Fedarko, M.W., et al., Visualizing 'omic feature rankings and log-ratios using Qurro. NAR Genom Bioinform, 2020. 2(2): p. Iqaa023.

39. Arumugam, M., et al., Enterotypes of the human gut microbiome. Nature, 2011. 473(7346): p. 174-80.

40. Holmes, I., K. Harris, and C. Quince, Dirichlet multinomial mixtures: generative models for microbial metagenomics. PLoS One, 2012. 7(2): p. e30126.

787 41. Blei, D., Ng AY, Jordan MI, Latent Dirichlet allocation. J Mach Learn Res, 2003. 3: p. 993-1022.

42. Sankaran, K. and S.P. Holmes, Latent variable modeling for the microbiome. Biostatistics, 2019. 20(4): p. 599-614.

791 43. Liao, T., et al., tmap: an integrative framework based on topological data analysis for population-scale microbiome stratification and association studies. Genome Biol, 2019. 20(1): p. 293.

44. Vandeputte, D., et al., Quantitative microbiome profiling links gut community variation to microbial load. Nature, 2017. 551(7681): p. 507-511. 
796 45. Vieira-Silva, S., et al., Statin therapy is associated with lower prevalence of gut microbiota dysbiosis. Nature, 2020. 581(7808): p. 310-315.

46. Breuninger, T.A., et al., Associations between habitual diet, metabolic disease, and the gut microbiota using latent Dirichlet allocation. Microbiome, 2021. 9(1): p. 61.

800 47. Hosoda, S., et al., Revealing the microbial assemblage structure in the human gut

48. Sommeria-Klein, G., et al., Latent Dirichlet Allocation reveals spatial and taxonomic structure in a DNA-based census of soil biodiversity from a tropical forest. Mol Ecol

49. Romano, S., et al., Meta-analysis of the Parkinson's disease gut microbiome suggests alterations linked to intestinal inflammation. NPJ Parkinsons Dis, 2021. 7(1): p. 27.

50. Singh, G., M'emoli, F., and Carlsson, G. E., Topo-logical methods for the analysis of

51. Meehan, C.J. and R.G. Beiko, A phylogenomic view of ecological specialization in the Lachnospiraceae, a family of digestive tract-associated bacteria. Genome Biol Evol,

52. Vacca, M., et al., The Controversial Role of Human Gut Lachnospiraceae. Microorganisms, 2020. 8(4).

814 53. Christensen, L., et al., Microbial enterotypes beyond genus level: Bacteroides species as

54. De Filippis, F., et al., Distinct Genetic and Functional Traits of Human Intestinal Prevotella copri Strains Are Associated with Different Habitual Diets. Cell Host Microbe, 2019. 25(3): p. 444-453 e3.

55. De Filippis, F., et al., Unusual sub-genus associations of faecal Prevotella and Bacteroides with specific dietary patterns. Microbiome, 2016. 4(1): p. 57. 
822 56. Gorvitovskaia, A., S.P. Holmes, and S.M. Huse, Interpreting Prevotella and Bacteroides as biomarkers of diet and lifestyle. Microbiome, 2016. 4: p. 15.

824 57. Levy, R., et al., Longitudinal analysis reveals transition barriers between dominant ecological states in the gut microbiome. Proc Natl Acad Sci U S A, 2020. 117(24): p. 13839-13845.

827 58. Wu, G.D., et al., Linking long-term dietary patterns with gut microbial enterotypes. Science, 2011. 334(6052): p. 105-8.

829 59. Wang, D.D., et al., The gut microbiome modulates the protective association between a Mediterranean diet and cardiometabolic disease risk. Nat Med, 2021. 27(2): p. 333-343.

831 60. Ley, R.E., Gut microbiota in 2015: Prevotella in the gut: choose carefully. Nat Rev Gastroenterol Hepatol, 2016. 13(2): p. 69-70.

833 61. Henderson, G., et al., Improved taxonomic assignment of rumen bacterial 16S rRNA sequences using a revised SILVA taxonomic framework. PeerJ, 2019. 7: p. e6496.

62. Tett, A., et al., The Prevotella copri Complex Comprises Four Distinct Clades 679 e7.

838 63. Tett, A., et al., Prevotella diversity, niches and interactions with the human host. Nat Rev Microbiol, 2021.

840 64. Scher, J.U., et al., Expansion of intestinal Prevotella copri correlates with enhanced

842 65. Albert, M.S., et al., The diagnosis of mild cognitive impairment due to Alzheimer's susceptibility to arthritis. Elife, 2013. 2: p. e01202.

846 66. McKhann, G.M., et al., The diagnosis of dementia due to Alzheimer's disease: recommendations from the National Institute on Aging-Alzheimer's Association 
workgroups on diagnostic guidelines for Alzheimer's disease. Alzheimers Dement, 2011. 7(3): p. 263-9.

67. Wallin, A.K., et al., CSF biomarkers predict a more malignant outcome in Alzheimer

68. Demircan, T., et al., Experimentally induced metamorphosis in highly regenerative axolotl (ambystoma mexicanum) under constant diet restructures microbiota. Sci Rep,

69. Martin, M., Cutadapt removes adapter sequences from high-throughput sequencing reads. EMBnet J, 2011. 17:10-12.

70. Weber, N., et al., Nephele: a cloud platform for simplified, standardized and reproducible

71. Davis, N.M., et al., Simple statistical identification and removal of contaminant sequences in marker-gene and metagenomics data. Microbiome, 2018. 6(1): p. 226.

861 72. R Core Team (2020).R: A language and environment for statistical computing. $R$ Foundation for Statistical Computing, Vienna, Austria. URL https://www.R-project.org/. 2020.

73. McMurdie, P.J. and S. Holmes, phyloseq: an $R$ package for reproducible interactive

74. Ritchie, M.E., et al., limma powers differential expression analyses for RNA-sequencing and microarray studies. Nucleic Acids Res, 2015. 43(7): p. e47.

868 75. Clarke, K.R.a.G., R. N., PRIMER v7: User Manual/Tutorial. PRIMER-E Plymouth. 1993.

869 76. Vazquez-Baeza, Y., et al., EMPeror: a tool for visualizing high-throughput microbial community data. Gigascience, 2013. 2(1): p. 16.

871 77. Himel Mallick, L.J.M., Ali Rahnavard, Siyuan Ma,Yancong Zhang, Long H. Nguyen1, 

omics Studies, in bioRxiv. 2021.

876 78. Ding, T. and P.D. Schloss, Dynamics and associations of microbial community types

877 across the human body. Nature, 2014. 509(7500): p. 357-60.

878 79. Yan, J., et al., MetaTopics: an integration tool to analyze microbial community profile by topic model. BMC Genomics, 2017. 18(Suppl 1): p. 962.

880

881 
Table 1. Demographic characteristics of the participants in the cohort

\begin{tabular}{|c|c|c|c|}
\hline & C & $\mathrm{MCl}$ & AD \\
\hline$n,(N=125)$ & 51 & 27 & 47 \\
\hline Sex (Female, N\%) & $45 \%(23 / 51)$ & $41 \%(11 / 27)$ & $49 \%(23 / 47)$ \\
\hline Age (years, mean \pm SDEV) & $67 \pm 5.3$ & $69.2 \pm 6.4$ & $71.4 \pm 5.1$ \\
\hline Education (Years) & $7.2 \pm 4.1$ & $10.4 \pm 5.2$ & $4.4 \pm 4.1$ \\
\hline MMSE & $27.1 \pm 1.7$ & $25.4 \pm 2.7$ & $16.9 \pm 5.7$ \\
\hline \multicolumn{4}{|l|}{ CDR } \\
\hline 0 & $100 \%$ & $0 \%$ & 0 \\
\hline 0.5 & 0 & $100 \%(27 / 27)$ & $29.8 \%(14 / 47)$ \\
\hline 1 & 0 & 0 & $31.9 \%(15 / 47)$ \\
\hline 2 & 0 & 0 & $29.8 \%(14 / 47)$ \\
\hline 3 & 0 & 0 & $8.5 \%(4 / 47)$ \\
\hline Aß1-42/P-Tau (pg/mL) & NA & NA & $5.97 \pm 3.7(n=14)$ \\
\hline A $\beta 1-42 / T-T a u(p g / m L)$ & NA & NA & $0.91 \pm 0.6(n=14)$ \\
\hline \multicolumn{4}{|l|}{ Medications } \\
\hline AA & NA & $37 \%(10 / 27)$ & $27.6 \%(13 / 47)$ \\
\hline ADd & NA & $81 \%(22 / 27)$ & $87 \%(41 / 47)$ \\
\hline Adep & NA & $66.7 \%(18 / 27)$ & $27.6 \%(13 / 47)$ \\
\hline $\mathrm{AE}$ & NA & $18.5 \%(5 / 27)$ & $8.5 \%(4 / 47)$ \\
\hline Aht & NA & $48 \%(13 / 27)$ & $29.8 \%(14 / 47)$ \\
\hline Apsik & NA & $11.1 \%(3 / 27)$ & $21.2 \%(10 / 47)$ \\
\hline Adiab & NA & $29.6 \%(8 / 27)$ & $19.1 \%(9 / 47)$ \\
\hline PP & NA & $7.4 \%(2 / 27)$ & $6.3 \%(3 / 47)$ \\
\hline
\end{tabular}

C: Control group, $\mathrm{MCl}$ : Mild Cognitive Impairment group; AD: Alzheimer's Disease group; MMSE: Mini-Mental State Exam (MMSE); CDR:Clinical Dementia Rating. AA:Antiaggregant; ADd:AD-treatment; Adep: Antidepressant; AE:Antiepileptic; Aht:Antihypertansive; Apsik: Antipychotic; Adiab:Antidiabetic; PP: Proton-pump inhibitor 
- Firmicutes

Bacteroidetes

- Proteobacteria

Verrucomicrob

- Actinobacteria

- Tenericutes

Elusimicrobia

Lentisphaerae

- Fusobacteria

- Synergistetes

- Spirocheotes

Epsilonbactera
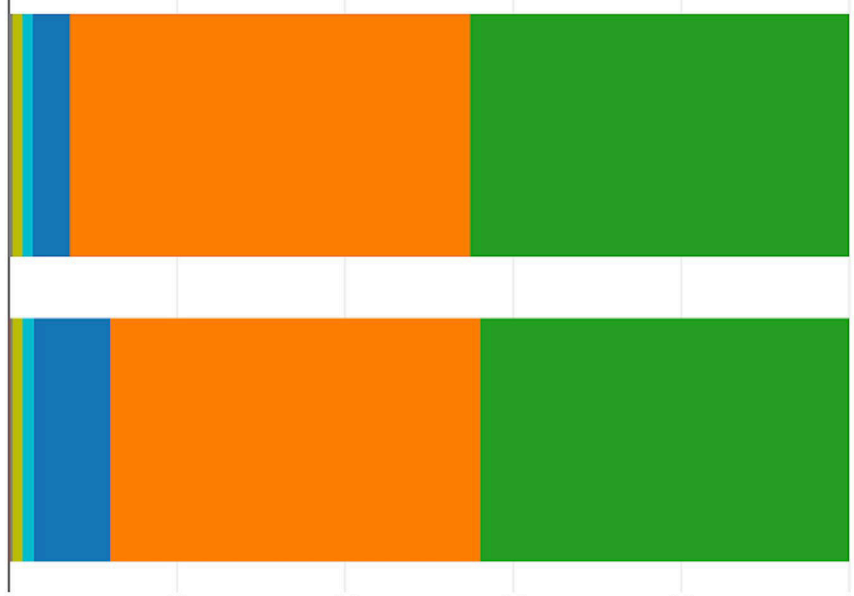

0.2

0.6

0.8

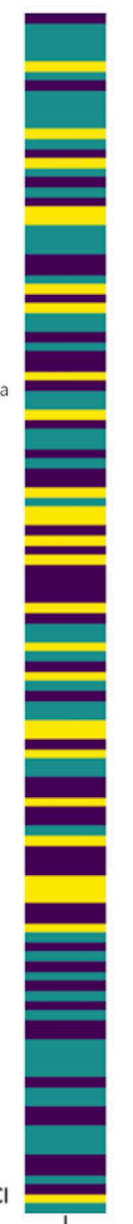

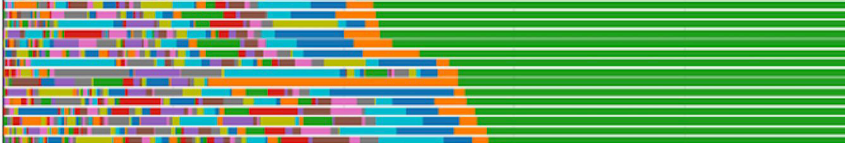

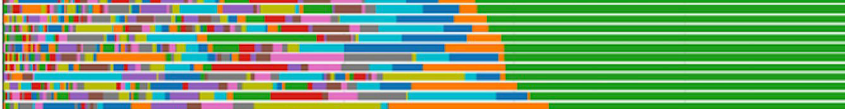

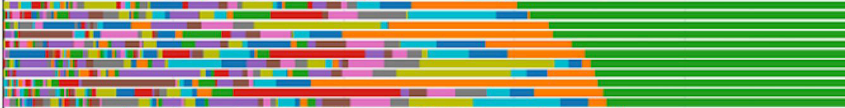

Pine

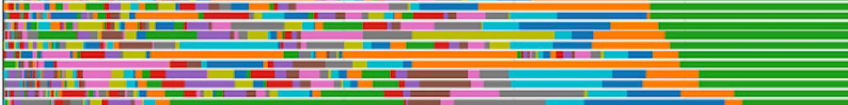

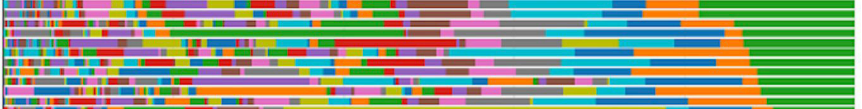

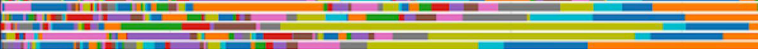

IIII

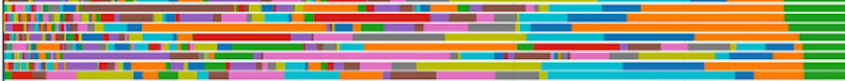

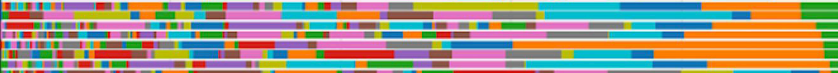

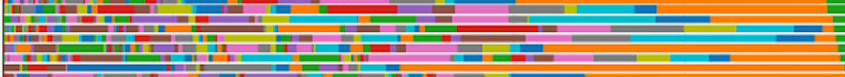

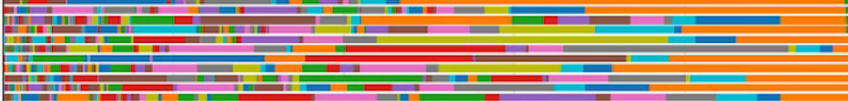

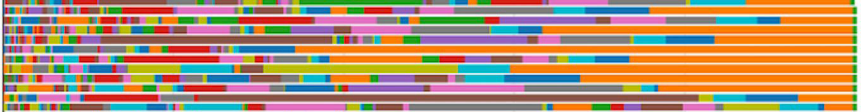

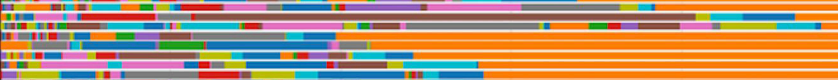

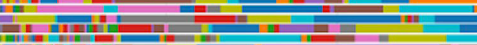

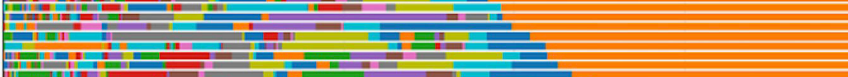

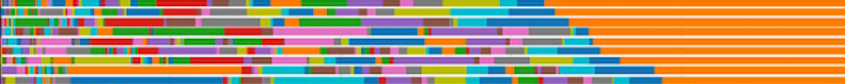

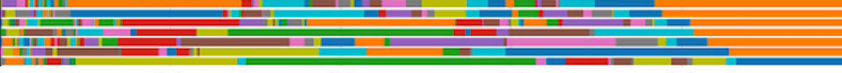
$0_{0}^{20}$
Prevotella_9

Bacteroides

- Faecalibacterium

Lachnospiraceae_unclassified

Escherichia/Shigella

Ruminococcaceae_unclassified

Ruminococcaceae_UCG-002

Blautia

Alistipes

$\square$ Dialister

Agathobacter

- Subdoligranulum

Parabacteroides

Roseburia

Lachnospira

Ruminococcus_2

- Ruminococcaceae_UCG-014

- Ruminococcus_1

Lachnospiraceae_NK4A136_group

Barnesiella

akkermansia

Succinivibrio

- Coprococcus_2

- Alloprevotella

Dorea

- Sutterella

- Lactobacillus

- Muribaculaceae_unclassified

- Phascolarctobacterium

口 Bifidobacterium 
A

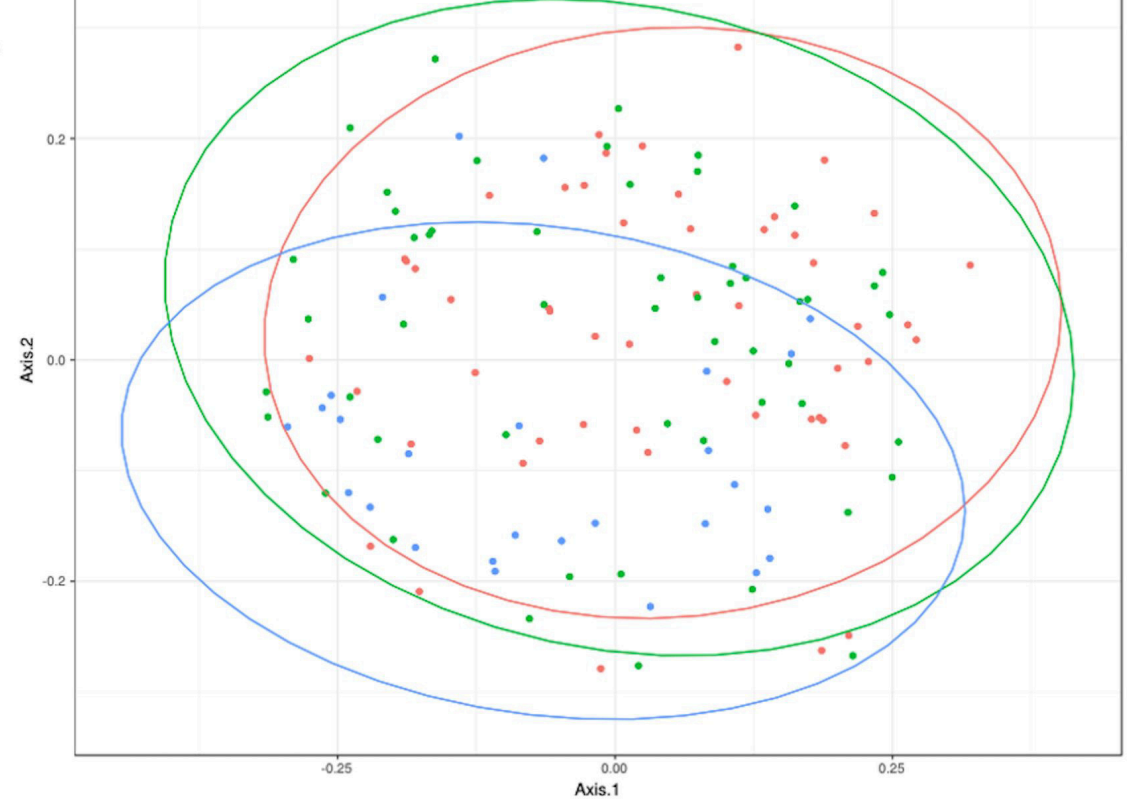

C

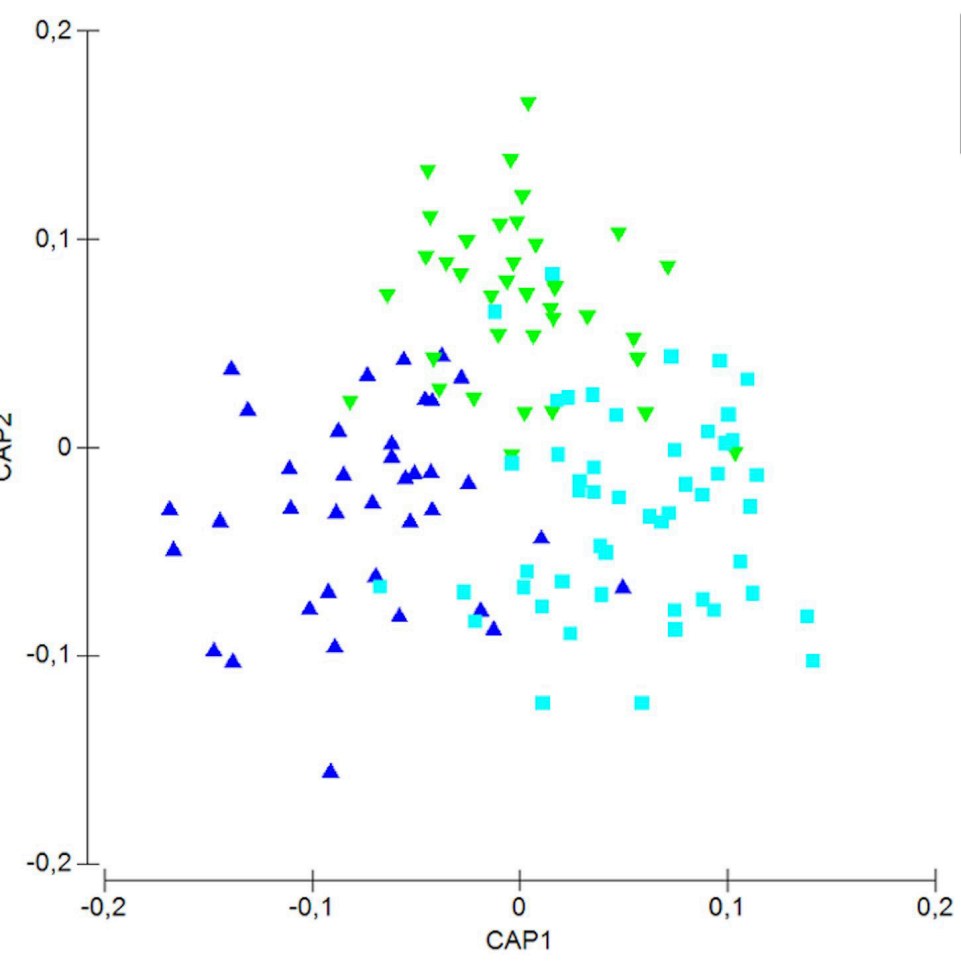

\begin{tabular}{|l|}
\hline Group \\
$\triangle \mathrm{AD}$ \\
$\nabla \mathrm{MCl}$ \\
$\mathrm{C} \mathrm{C}$ \\
\hline
\end{tabular}
B

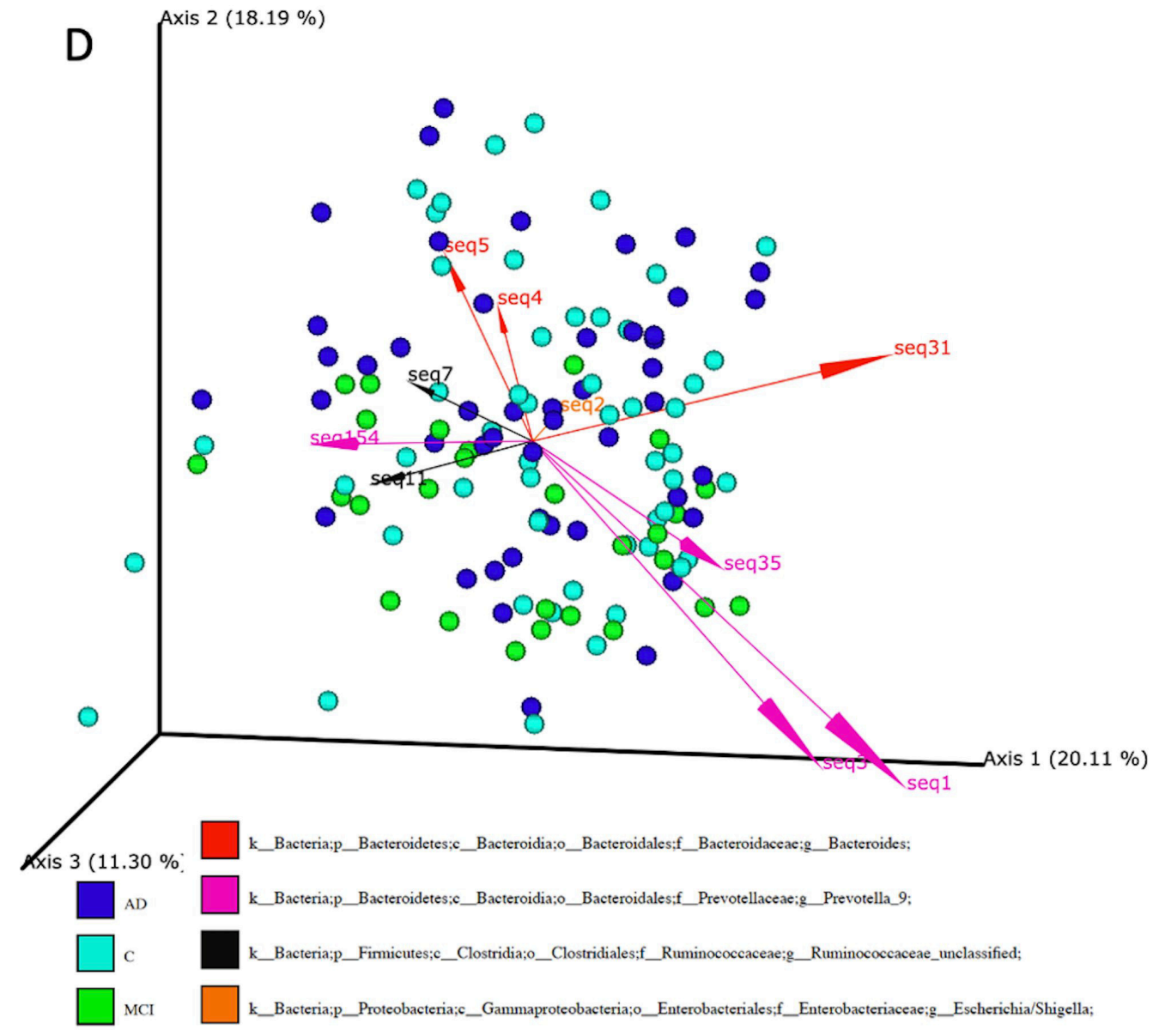




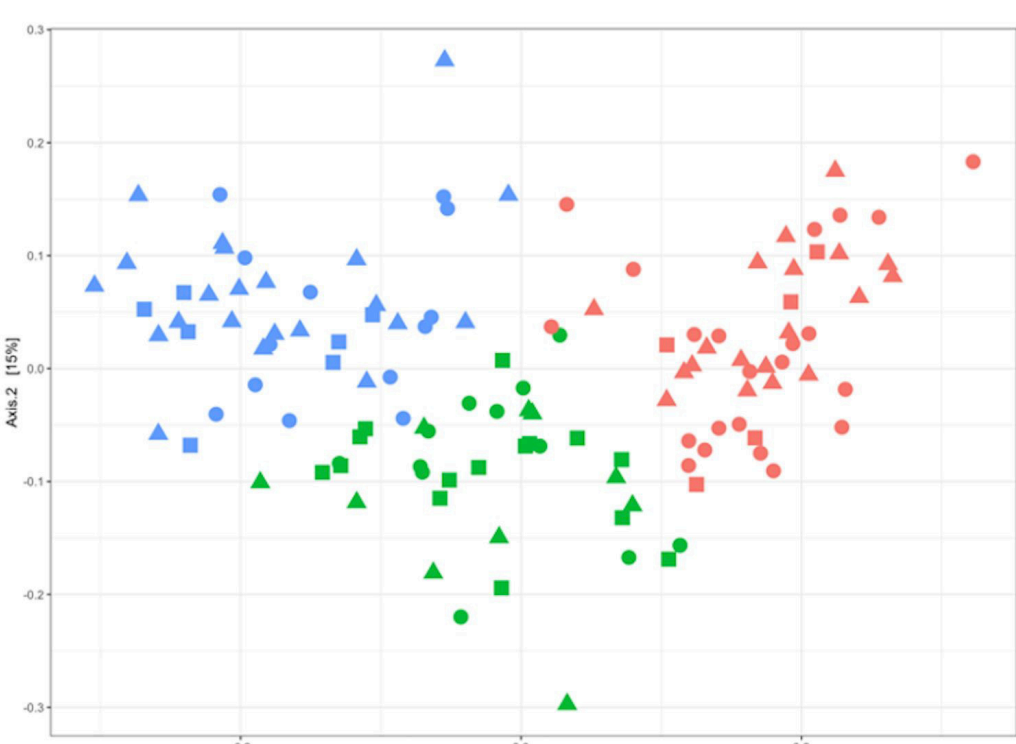

Axis. 1 [43.4\%]
C

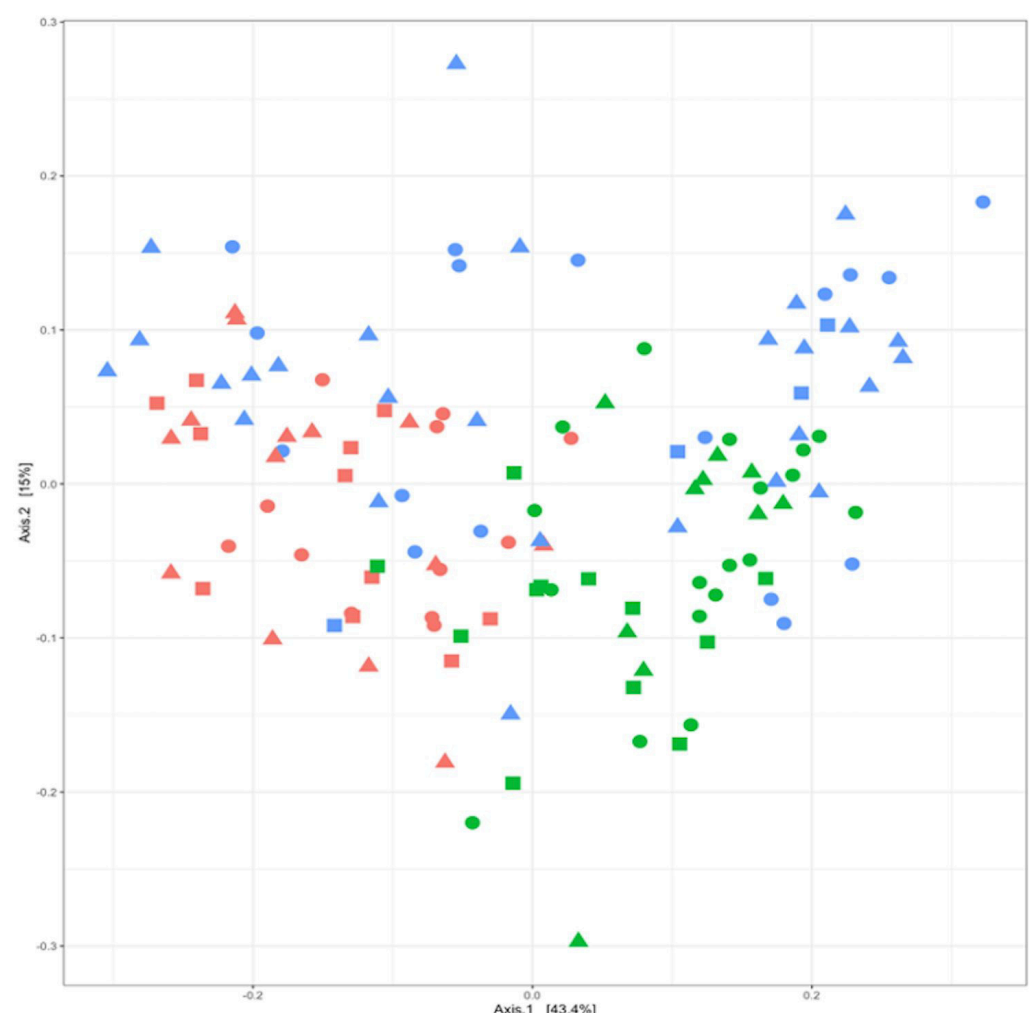

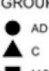

PAM cluster

$\because 8$

and

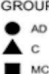

Dan cusers

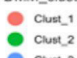

B

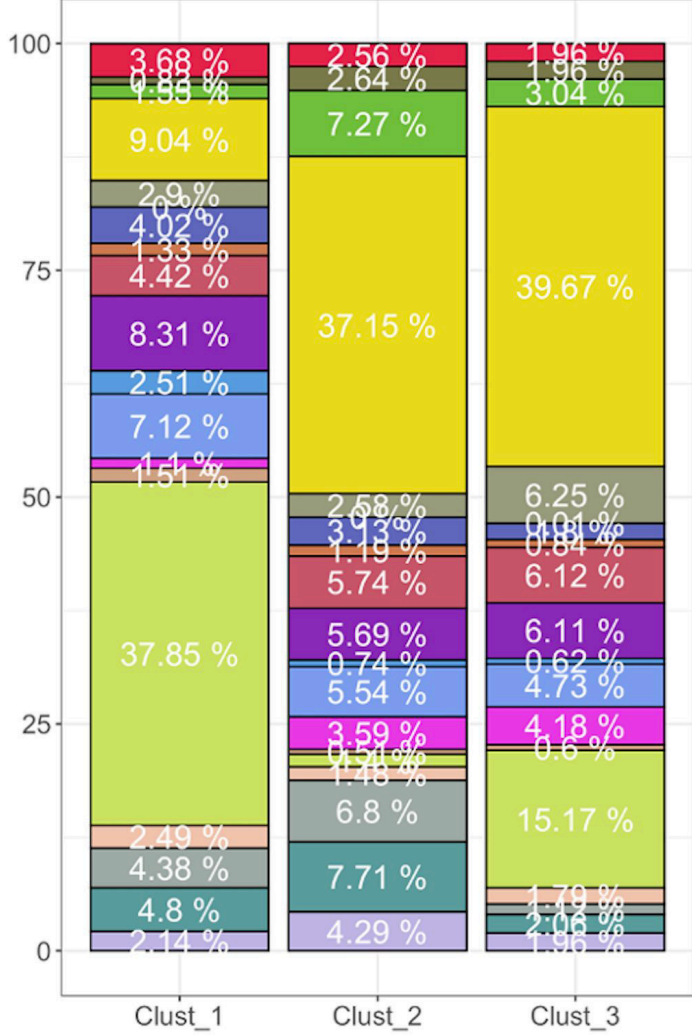

D

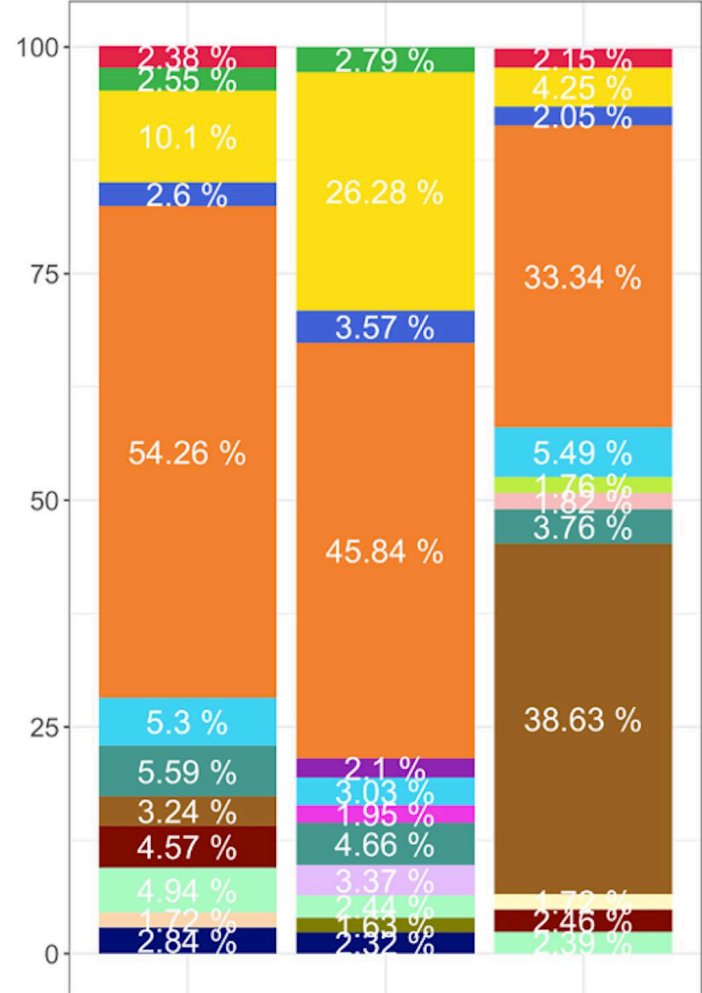

Cluster1
Genus

Agathobacter

Alistipes

Bacteroides

Blautia

Others

Escherichia/Shigella

Faecalibacterium

Lachnospira

Lachnospiraceae_NK4A136_group Lachnospiraceae_Unclassified Parabacteroides

Prevotella_9

Roseburia

Ruminococcaceae_UCG-002

Ruminococcaceae Unclassified

Ruminococcus_1

Ruminococcus_2 

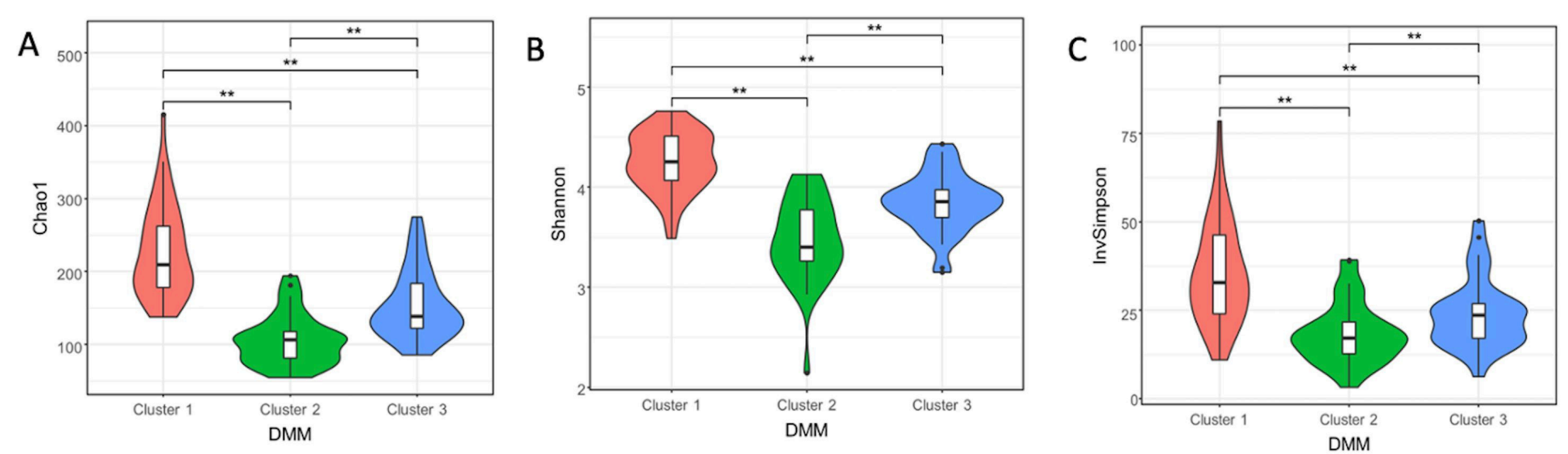

D
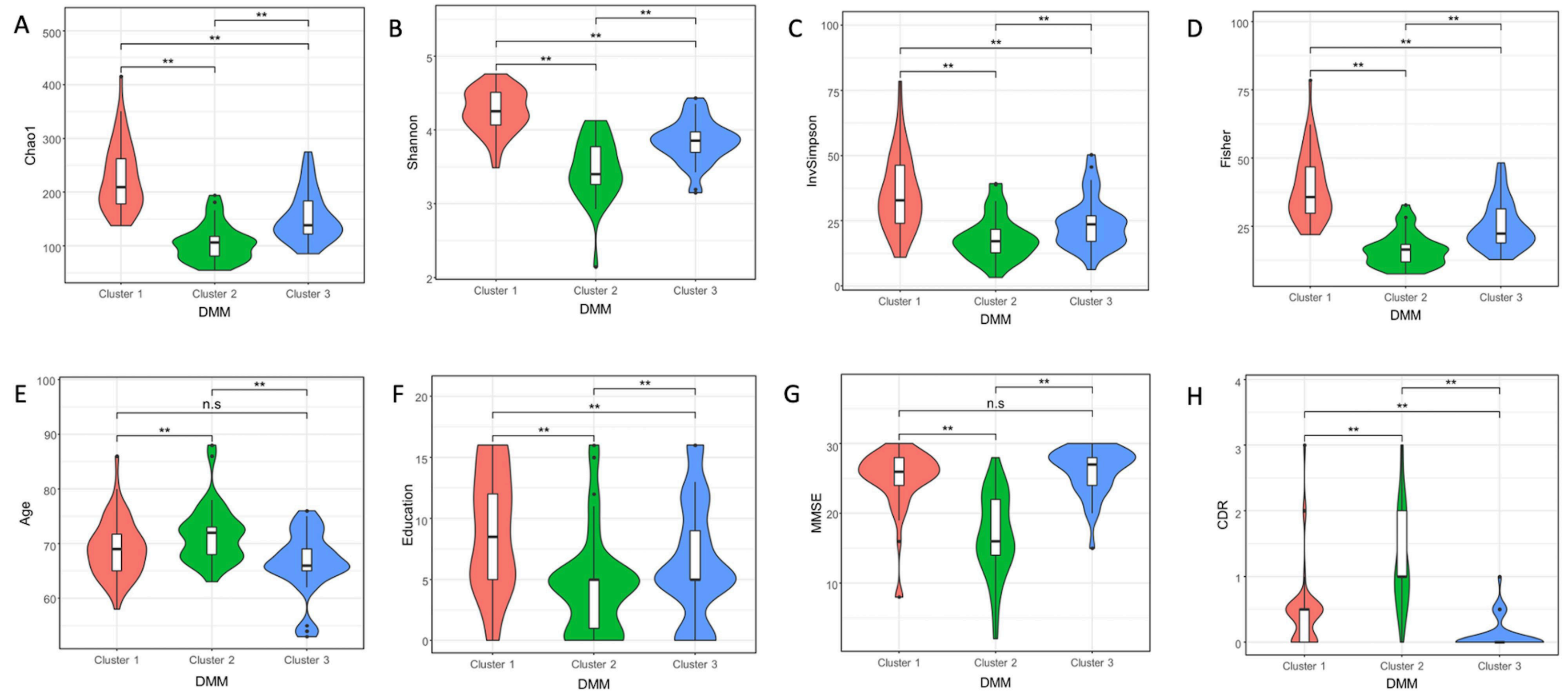

H

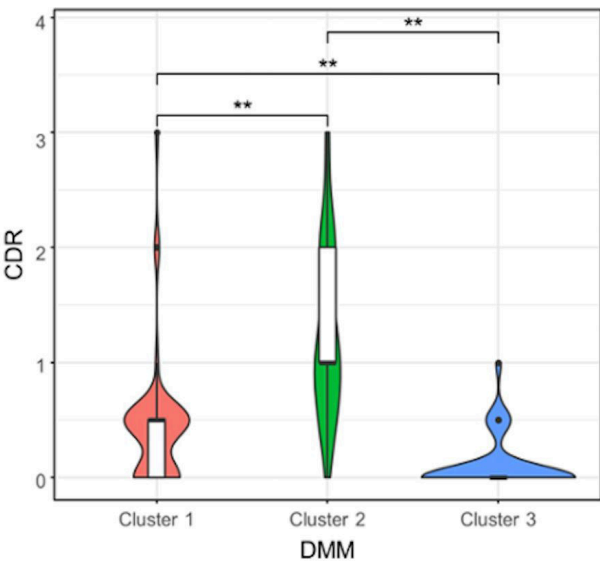


A $\quad \begin{aligned} & \text { topic vs. bacteria probability distributio } \\ & \text { (points probability }<0.05 \text { filtered) }\end{aligned}$

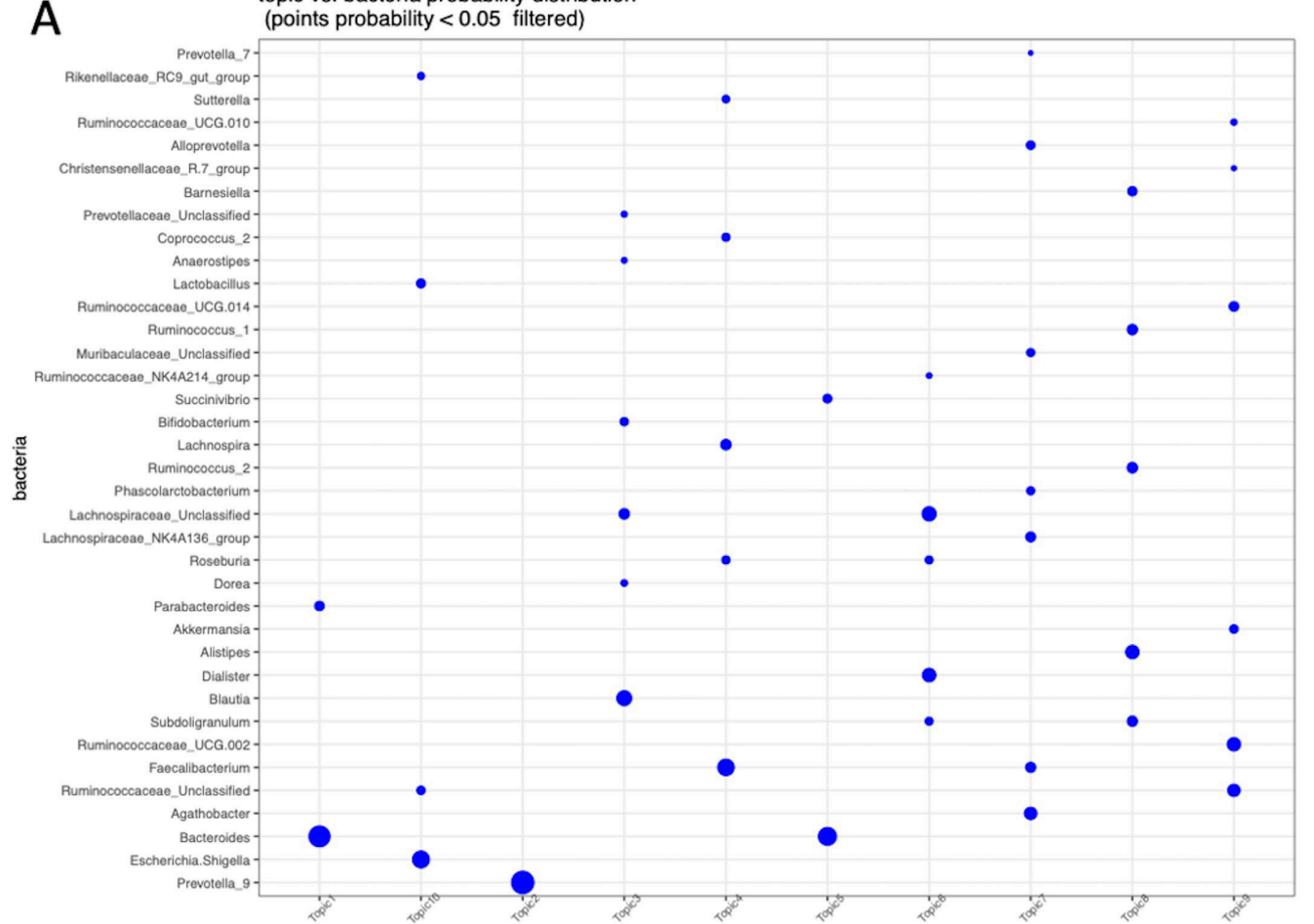

B

topic 


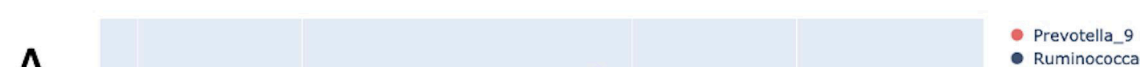

: Prevotella__a
: Ruminincoccaceae_unclassfified
Bacterodes

: Bacteroides Lachospiraceae_NK4A136_group

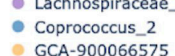

- Lachnospiraceae_unclassified
- Ruminococcaceae_UCG-002
- GCA-9000066225

GCA-900066225 Christensenellaceae_R-7__roup

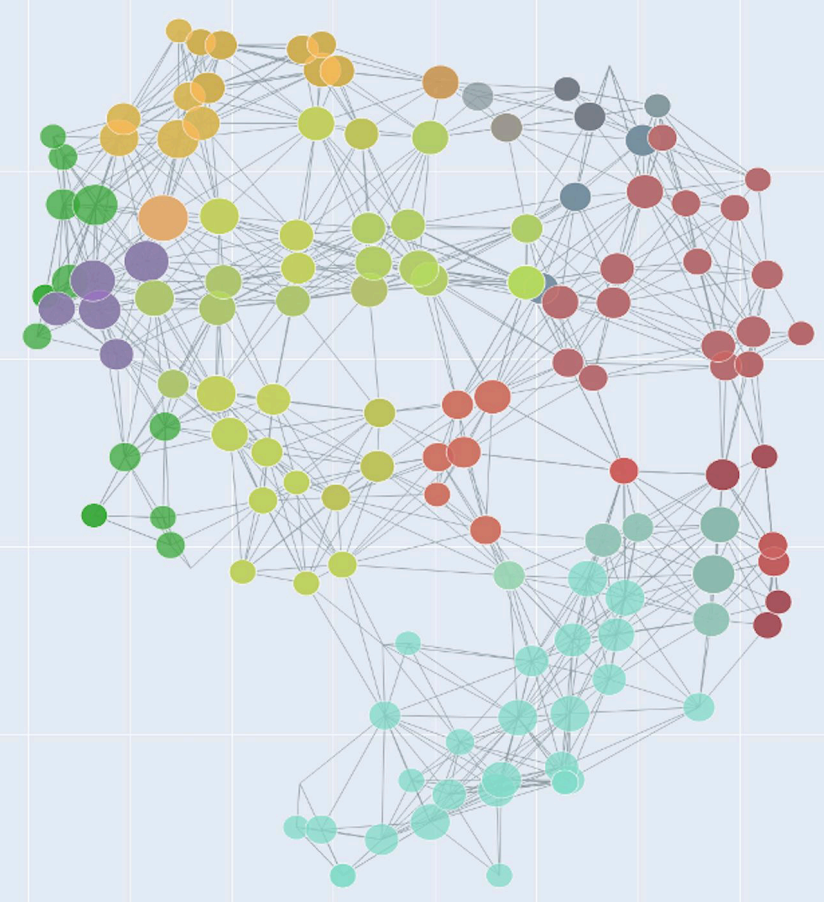

- Ruminococcaceae_unclassifie

- Prevotella_9

- Lachnospiraceae_unclassified

- Mollicutes_RF39_unclassified_unclassified

- Ruminococcaceae_UCG-002

- Bacteroides

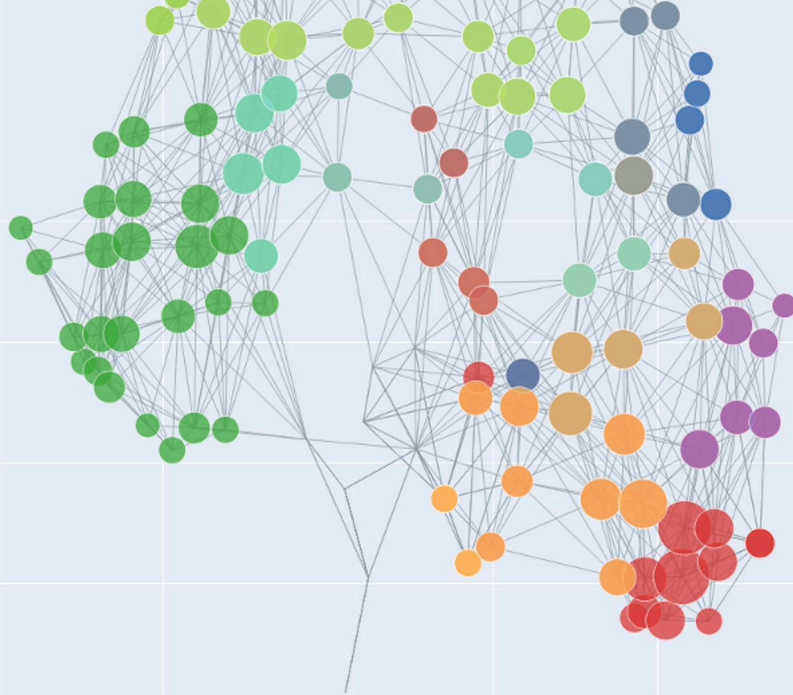




\section{AUC for ROC}

A

ه্ব

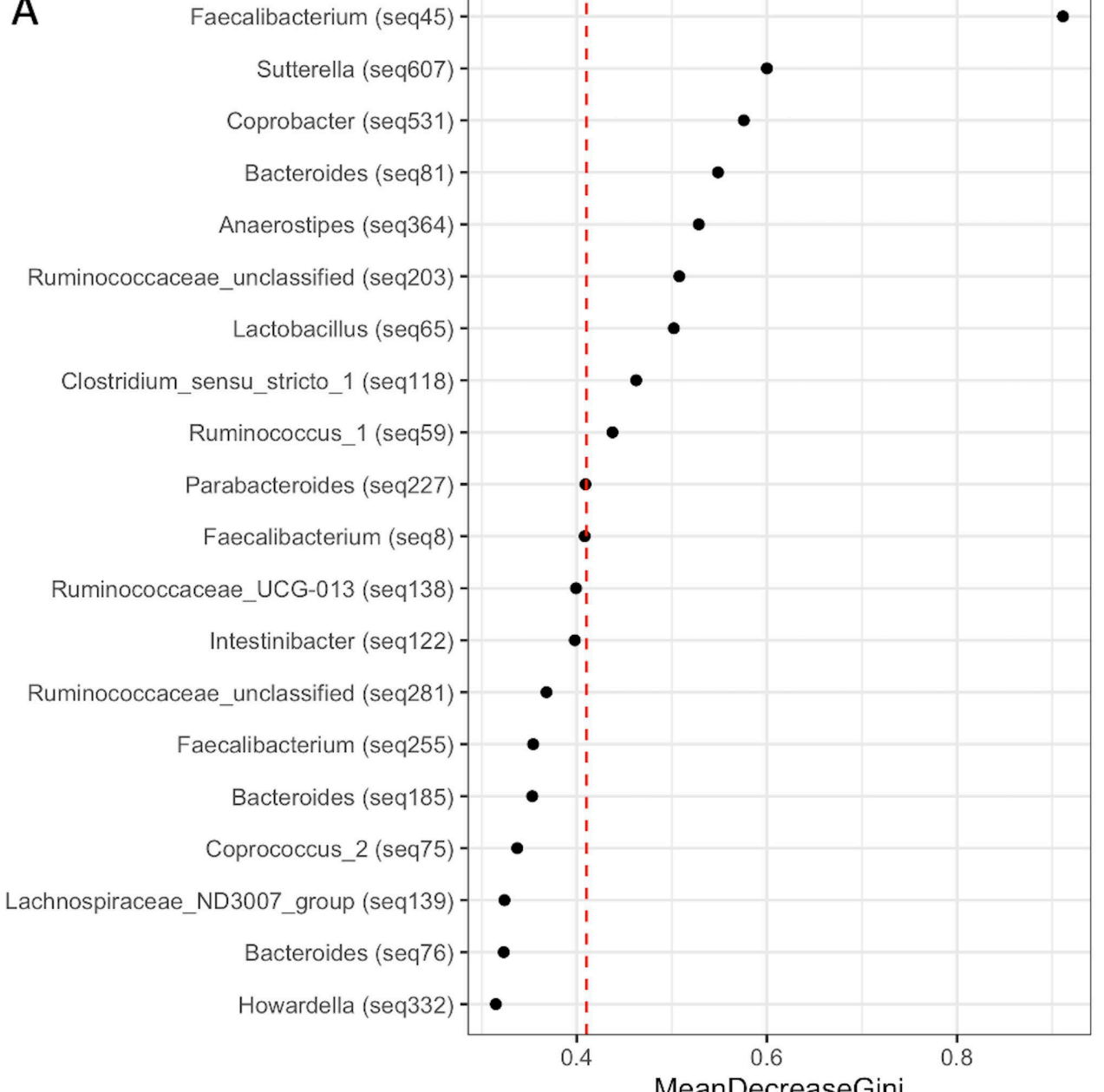

MeanDecreaseGini

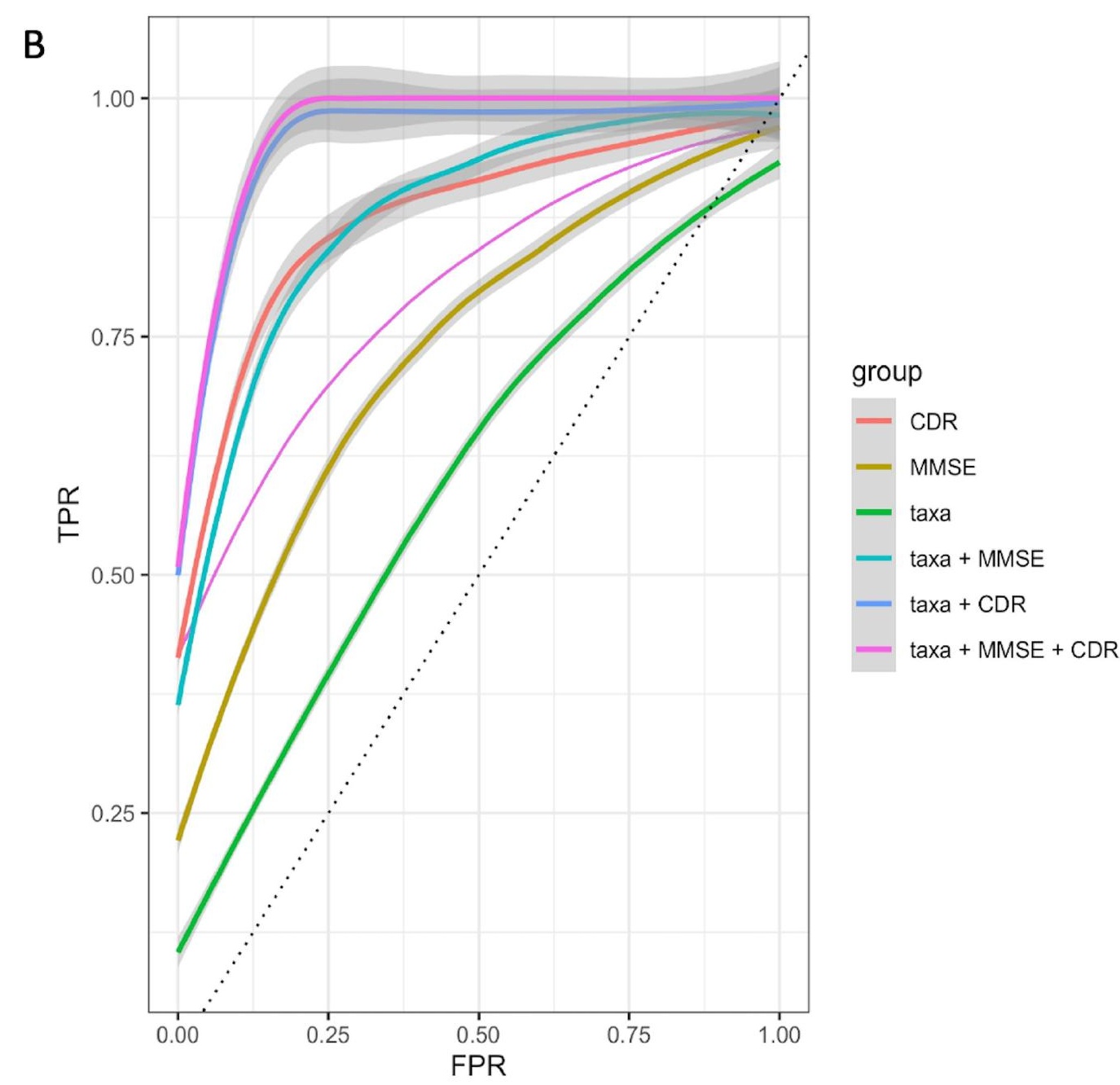

B 